\title{
EQUATIONAL BASES AND NONMODULAR LATTICE VARIETIES $\left({ }^{1}\right)$
}

BY

\author{
RALPH MCKENZIE
}

ABSTRACT. This paper is focused on equational theories and equationally defined varieties of lattices which are not assumed to be modular. It contains both an elementary introduction to the subject and a survey of open problems and recent work. The concept of a "splitting" of the lattice of lattice theories is defined here for the first time in print. These splittings are shown to correspond bi-uniquely with certain finite lattices, called "splitting lattices". The problems of recognizing whether a given finite lattice is a splitting lattice, whether it can be embedded into a free lattice, and whether a given interval in a free lattice is atomic are shown to be closely related and algorithmically solvable. Finitely generated projective lattices are characterized as being those finitely generated lattices that can be embedded into a free lattice.

Introduction. What we do in this paper is fairly described by the phrase "equational model theory of lattices." We take this in a broad sense, as the study of lattices and their equational theories, with particular emphasis on finding connections relating algebraic properties of lattices to various properties of the ir theories. Since many interesting properties of lattice theories can be defined abstractly, purely by reference to the lattice of all such theories, we are naturally led to investigate the structure of $\mathfrak{F}_{l}$, the lattice of all equational theories of lattices.

Recent studies in this largely unexplored field have been greatly stimulated by a paper of Bjarni Jónsson [6]. The central result of that paper ([6, Corollary 3.2], stated below as Lemma 1.4) has already found a number of applications, in the original paper as well as in [1], [7], [9] and the present paper.

Broadly speaking, Jónsson's result tells us that the cords binding lattices to the ir theories are much more tightly drawn than one would expect on the basis of experience with other equationally defined classes of algebras, such as groups and rings. The happy consequences of this fact, both for the algebraic and the equational study of lattices, will hardly be exhausted by the present paper; and we expect other researchers to take up the challenge. To that end, we have given

Received by the editors March 5, 1969.

AMS (MOS) subject classifications (1970). Primary 08A15, 06A20, 02G05, $02 \mathrm{G} 15$.

Key words and phrases. Lattice varieties, equational bases, irreducible equations, projective lattices, splitting lattices, atomic intervals of a free lattice, algorithms, finitely based equational theories, Jónsson theorem for congruence distributive varieties.

$\left({ }^{1}\right)$ Research and writing supported by U. S. National Science Foundation grant GP. 7578. 
explicit formulation to eleven unsolved problems, and many others are scattered through the text.

The first section of our paper contains preliminaries: basic concepts and notation, a summary of some recent results, and a formulation of the very useful facts from [6].

The central notion of the paper, which we analyze extensively, is the notion of a splitting of $\mathfrak{T}_{l}$, defined in $\S_{3}$. The splittings we have in mind are occasioned by those pairs of theories, $\langle\mathrm{H}, \Theta\rangle$, for which every lattice theory $\mathrm{K}$ belongs to just one of two intervals in $\mathfrak{T}_{l}: \mathrm{H} \leq \mathrm{K}$ or $\mathrm{K} \leq \Theta$.

Though perhaps it was never formulated in this way, it has long been known that the lattice $N_{5}$ (Diagram 2) gives rise to such a splitting of $\mathfrak{T}_{l}$ (or, more properly, the pair $\left\langle\mathrm{M}, \boldsymbol{\Theta} N_{5}\right\rangle$, where $\mathrm{M}$ is the theory of modular lattices). Since $N_{5}$ plays, in several ways, a generic part in these considerations, we have devoted $\$ 2$ to presenting a system of equations from which all equations of $N_{5}$ can be derived. (Our system of axioms for $N_{5}$ is a little stronger than that once given by Schutzenberger [10], thus demonstrating an error in his claim. For the details, see [3].)

In $\$ 4$, we show that the second term in each splitting pair is the theory of a finite, subdirectly irreducible lattice. And in $\$ 5$ we show how to characterize these lattices algebraically (Theorem 5.1). We call them splitting lattices. It turns out that the generation of splitting lattices is equivalent, in a sense, to the calculation of atomic quotients, $w_{0} \prec w_{1}$, in finitely generated free lattices (Theorem 5.4).

The two principal results of $\$ 5$, or rather the constructive methods implicit in the proofs and developed in $\$ 6$, yield some surprising corollaries. For instance, we obtain an algorithm for checking whether one word covers another in a free lattice (Theorem 6.2); and an algorithm for deciding whether a finite lattice can be embedded into a free lattice (Theorem 6.3. An essential portion of the argument was supplied by Jónsson).

The paper closes with a series of instructive examples ( $\$ 7$ ); and a discussion of the relation of covering in the lattice of theories (\$8), giving further applications of the methods developed earlier on, and a number of problems.

It is a pleasure to acknowledge the large contribution that Bjarni Jónsson has made to this paper, through his published writings and in conversations with the author; and the significant debt that I owe to Alfred Tarski and to my student, Alan Kostinsky, for their patience and good advice.

1. Preliminaries. Our treatment of the basic concepts of equational logic, and of the algebraic theory of lattices, is adapted from Tarski [11] and Birkhoff [2], respectively. Our notation differs, in a few details, from the notation of those authors. A fixed equational language with two binary operation symbols, + and $\cdot$, 
is required. We call it the basic equational language of lattices. All the equations and theories encountered below are assumed to be defined in this language.

A lattice is an algebraic system

$$
A=\langle|A|,+, \cdot\rangle
$$

satisfying identically the following equations of the basic language:

$$
\begin{array}{lll}
\lambda_{1} \cdot x \cdot y=y \cdot x, & \lambda_{2} \cdot x+y=y+x ; \\
\lambda_{3} \cdot x \cdot(y \cdot z)=(x \cdot y) \cdot z, & \lambda_{4} \cdot x+(y+z)=(x+y)+z ; \\
\lambda_{5} \cdot x \cdot(x+y)=x, & \lambda_{6} \cdot x+x \cdot y=x .
\end{array}
$$

Lattice theory is the set of all equations which can be derived (using the operations of substitution and replacement of equals by equals) from $\lambda_{1}-\lambda_{6}$. We shall denote it by $\Lambda$.

Throughout the paper, we treat only equational theories $\Theta$ extending $\Lambda$. They can all be obtained in the following way. We take an arbitrary set of equations, $\Sigma$, and form the theory $\Theta=\boldsymbol{\Theta}_{l}[\Sigma]$ consisting of all equations derivable from $\Lambda \cup$ $\Sigma$. We call $\Sigma$ a base for $\Theta$ (relative to $\Lambda$ ). If $\Sigma$ is a finite set $\left\{\epsilon_{0}, \epsilon_{1}, \ldots, \epsilon_{\mu}\right\}$, the resulting theory may be denoted by $\boldsymbol{\Theta}_{l}\left[\epsilon_{0}, \epsilon_{1}, \ldots, \epsilon_{\mu}\right]$.

The collection of all theories containing $\Lambda$, ordered by set-inclusion, forms a lattice

$$
\mathfrak{T}_{l}=\left\langle\mathbf{T}_{l},+, \cdot\right\rangle
$$

whose operations are defined by the rules

$$
\Theta_{0} \cdot \Theta_{1}=\Theta_{0} \cap \Theta_{1}, \quad \Theta_{0}+\Theta_{1}=\Theta_{l}\left[\Theta_{0} \cup \Theta_{1}\right] .
$$

Each lattice $L$ determines a theory $\boldsymbol{Q}$, consisting of all equations valid in $L$; and conversely, every theory $\Theta \in \mathbf{T}_{l}$ is the theory of some lattice $L$. [Given $\Theta$, we can take for $L$ any $\Theta$-free lattice, $F L(\Theta, X)$, on an infinite set $X$ of free generators; for example, the algebra of terms of the basic language, reduced by the relation $\sim_{\theta}$ of $\Theta$-equivalence: $\sigma \sim_{\theta} \tau$ iff $\sigma=\tau \in \Theta$.] Thus, $\mathscr{T}_{l}$ is appropriately called the lattice of equational theories of lattices.

We use the same symbolism for the theory of a class of lattices: by definition,

$$
\boldsymbol{\Theta K}=\bigcap\{\boldsymbol{\Theta} L: L \in \mathcal{K}\} \text {. }
$$

In case $\mathcal{K}$ is empty, the convention is $\mathbf{Q}=\Omega$, the largest theory.

The most important classes, from our standpoint, are the varieties (equational classes, primitive classes). A lattice variety is a class 0 of lattices which, for some $\Theta \in \mathbf{T}_{l}$, is defined by the condition

$$
L \in \mathcal{O} \Leftrightarrow \Theta \leq \boldsymbol{\Theta} \text {. }
$$


We write $\mathcal{O}=\mathcal{O} \Theta$. We have already seen how every equation or set of equations, likewise every lattice or class of lattices, determines a member of $\mathbf{T}_{l}$; thus each such entity determines a lattice variety. The corresponding variety will always be denoted by prefixing the symbol $\mathcal{O}$.

Two familiar theorems of Birkhoff and Tarski have concise symbolic formulations in this context. Assume that $\Theta_{0}, \Theta_{1} \in \mathbf{T}_{l}$, that $\mathcal{K}$ is a class of lattices, and that $\mathcal{O}$ is a lattice variety. Then we have

$$
\begin{aligned}
& \Theta_{0} \leq \Theta_{1} \Leftrightarrow \Theta_{1} \subseteq \Theta_{0} ; \\
& \Theta \mathcal{O} \Theta_{0}=\Theta_{0}, \vartheta \Theta \vartheta=0 \text {; } \\
& O K=\text { HSPK. }
\end{aligned}
$$

The first three relations above express a basic duality between theories and varieties; the last relation contains the fact that every lattice in $\mathcal{O K}$ can be constructed algebraically as a homomorphic image of a sublattice of a direct product of lattices belonging to $K$.

We will use standard notation for the intervals and the covering relation of any lattice under consideration, and the notation ${ }^{*}(X)$ for the cardinal number of a set $X$. Thus

$$
\begin{aligned}
{[x, y] } & =\{z: x \leq z \leq y\}, \quad[x, y)=\{z: x \leq z<y\} ; \\
& x<y(y \text { covers } x) \Leftrightarrow \#([x, y])=2 .
\end{aligned}
$$

Many equations can be most simply expressed as inclusions. The inclusion $\sigma \leq \tau$ denotes the equation $\sigma=\sigma \cdot \tau$ in the basic language. At times we will write $\sigma \leq_{\boldsymbol{\theta}} \tau$ to indicate that $\sigma \leq \tau \in \Theta$.

A natural notion of independence plays a role in the discussion of covering in $\mathfrak{F}_{l}(\S 8)$. A set of equations, $\Sigma$, is said to be independent (relative to $\Lambda$ ), or an independent base for $\boldsymbol{\Theta}_{l}[\Sigma]$, provided that $\boldsymbol{\Theta}_{l}\left[\Sigma^{\prime}\right]<\boldsymbol{\Theta}_{l}[\Sigma]$ for each proper subset $\Sigma^{\prime} \subset \Sigma$. We write $\nabla_{l} \Theta$ to denote the set of cardinalities of the independent bases of a theory $\Theta \in \mathrm{T}_{l .}{ }^{2}$ )

By reference to the corresponding theory, we can speak of the bases, and the independent bases, of a given lattice or class of lattices. For illustration, a theory, a lattice, or a class of lattices is said to be finitely based if it possesses a base composed of a finite number of equations. In [9, Theorem 2.1], it was proved that every finite lattice is finitely based; examples of nonfinitely based lattice varieties appear in [1] and [9].

We will state, in $\$ 8$, a number of problems involving the independent bases of theories; but the three lemmas below are immediate.

$\left(^{2}\right)$ For theories containing $\Lambda, \nabla_{l} \Theta$ usually gives more information about $\Theta$ than does Tarski's $\nabla \Theta[11, \S 4]$. A detailed comparison of these notions can be pieced together from [11, Theorem 8], the introduction to [9] and the remarks in this paper. 
Lemma 1.1. For every two equations $\epsilon_{0}, \epsilon_{1}$, there is an equation $\epsilon$ such that $\boldsymbol{\Theta}_{l}\left[\epsilon_{0}, \epsilon_{1}\right]=\boldsymbol{\Theta}_{l}[\epsilon]$.

Lemma 1.2. For each $\Theta \in \mathbf{T}_{l}$, one of the following statements is valid:

(i) $\Theta=\Lambda$ and $\nabla, \Theta=\{0\}$;

(ii) $\Theta$ is finitely based and either $\nabla_{l} \Theta=[1, \omega)$, or for some integer $\nu \geq 1$, $\nabla_{l} \Theta=[1, \nu]$

(iii) $\Theta$ is not finitely based and either $\nabla_{l} \Theta=\{\omega\}$ or $\nabla_{l} \Theta=0$.

The third lemma will provide a purely lattice theoretic definition of $\nabla_{l}$. We observe that the lattice of theories is a complete lattice; the least upper bound (or join) of an arbitrary set of theories $S$ is the theory $\boldsymbol{\theta}_{l}$ [US] for which we write VS. Finitely based theories $\Theta$ are also called compact because they are characterized within $\mathfrak{T}_{l}$ by the following property: $\Theta \leq V S$ implies the existence of a finite subset of $S, S^{\prime}$ with $\Theta \leq \bigvee S^{\prime}$.

Lemma 1.3. Assume that $\kappa \leq \omega$ and $\Theta \in \mathbf{T}_{l}$. An equivalent condition for $\kappa \in \nabla_{l} \Theta$ is the existence of a set of compact theories, $\mathrm{S}$, having the properties

(i) $\stackrel{F}{ }(\mathbf{S})=\kappa$,

(ii) $\bigvee \mathbf{S}=\Theta$,

(iii) $\bigvee \mathbf{S}^{\prime}<\Theta$ for each proper subset of $\mathbf{S}, \mathbf{S}^{\prime}$.

The proofs of the three lemmas are completely trivial. We only remark that in Lemma 1.1, assuming that $\epsilon_{\mu}=\sigma_{\mu}=\tau_{\mu}(\mu=0,1)$ and also that $\epsilon_{0}$ and $\epsilon_{1}$ have no variables in common, one can take for $\epsilon$ the equation $\sigma_{0} \cdot \sigma_{1}=\tau_{0} \cdot \tau_{1}$.

The values of $\nabla_{l}$ are known in only a few cases. Some examples given now provide additional basic information that will be useful later. At the top of the lattice $\mathfrak{T}_{l}$ is a group of four theories forming an invented $Y[6, \$ 4] . \Omega$ is the largest theory, based on the equation $x=y$. The next largest is $\Delta$, the theory of distributive lattices, based on the equation $x \cdot(y+z)=x \cdot y+x \cdot z$. Just below $\Delta$ are the theories of two lattices, $M_{3}$ and $N_{5}$ (Diagrams 1 and 2); every remaining theory is a subtheory of one of these two-this follows, of course, from the wellknown fact that every nondistributive lattice contains a sublattice isomorphic to either $M_{3}$ or $N_{5}$. From these facts one easily infers that $\nabla_{l} \Omega=\{1\}$ and $\nabla_{l} \Delta=$ $\{1,2\}$. For $\Theta M_{3}$ the problem is more difficult, but Jónsson [7] found that there are just three theories covered by $\Theta M_{3}$ and we infer from $\$ 8$ of this paper that $\nabla_{l} @ M_{3}=\{1,2,3\}$. Finally, $[1,16] \subseteq \nabla_{l} @ N_{5}(\$ 8)$, but we do not know whether the inclusion can be replaced by equality.

Among all the lattices of equational theories (of such well-known varieties of algebras as groups, rings, lattices, semigroups, etc.), $\mathfrak{T}_{l}$ itself has the simplest equational theory. In fact, $\mathfrak{I}_{l}=\Delta[6$, Corollary 4.1]. From this, and because every member of $\mathfrak{T}_{l}$ is a join of compact lattice theories, we actually have 


$$
\Theta \cdot \bigvee \mathbf{S}=\bigvee\{\Theta \cdot \Gamma: \Gamma \in \mathbf{S}\}
$$

whenever $\Theta \in \mathbf{T}_{l}$ and $\mathrm{S} \subseteq \mathrm{T}_{l}$. (The other infinite distributive law is false in $\mathfrak{S}_{l}$. Let $\Theta=\Theta M_{3}$ and $S=\left\{\Gamma: \Gamma \nless \bigotimes M_{3}\right\}$ and see Example 5.1.)

Despite the obvious simplification in calculations with theories afforded by these distributive laws, very little is known of the detailed structure of $\mathfrak{T}_{l}$. Kirby Baker [1, Theorem 3.1] discovered a complete sublattice of $\mathfrak{F}_{l}$ isomorphic to the boolean lattice $2^{\omega}$ of all subsets of $\omega$, and so we have $F\left(T_{l}\right)=2^{\boldsymbol{N}_{0}} \cdot\left(^{3}\right)$

From each equation $\epsilon$, we may obtain another equation $\epsilon^{d}$ by exchanging + and - at every appearance in $\epsilon$. From each theory $\Theta$, a dual theory is formed by collecting together the duals of equations in $\Theta: \Theta^{d}=\left\{\epsilon^{d}: \epsilon \in \Theta\right\}$. The familiar, special duality of lattice theory is expressed by the relation $\Lambda=\Lambda^{d}$. The duality map, restricted to $\mathrm{T}_{l}$, is an automorphism of $\stackrel{\mathfrak{F}}{l}_{l}$.

Problem 1. Are there any automorphisms of $\mathfrak{T}_{l}$ besides the identity map and the duality map, $d$ ?

To close this section, we turn from theories to varieties and state some fundamental results of $B$. Jónsson, which underlie many of the developments reported in this paper. $\left({ }^{4}\right)$ An algebra $A$ is said to be subdirectly irreducible (SI) provided that among the congruence relations of $A$, excluding the equality relation, there is a smallest. In other words, provided there exist two elements $a, b \in|A|$ with $a \neq b$, but $f a=f b$ for every homomorphism $f$ on $A$ which fails to be a monomorphism. A theorem of General Algebra, due to Birkhoff, states that every algebra is isomorphic to a subdirect product of its SI epimorphic images. Consequently, every variety is generated by its SI members, and $\mathcal{O A}$ is generated by the SI epimorphic images of $A$.

Now the central result of [6], when stated for lattices, is the following.

Lemma 1.4 [6, Corollary 3.2]. Let $\mathcal{K}$ be a class of lattices. Then every $S I$ lattice $L \in \mathcal{O K}$ is a bomomorpbic image of a sublattice of an ultraproduct of lattices from K. In symbols,

$$
L \in \mathbf{H S P}_{u} \text { K. }
$$

Using elementary properties of ultraproducts, Jónsson derived from this lemma a number of corollaries, which we briefly summarize.

For instance, given $\Theta_{0}, \Theta_{1} \in T_{l}, \mathcal{O} \Theta_{0} \cdot \Theta_{1}$ is generated by $\mathcal{O} \Theta_{0} \cup \mathcal{O}_{1}$; consequently, every SI member of $\mathcal{O} \Theta_{0} \cdot \Theta_{1}$ belongs to either $\mathcal{O} \Theta_{0}$ or $\mathcal{O}_{\Theta_{1}}$. Therefore, by the subdirect representation theorem, every lattice $L \in \mathcal{O}_{0} \cdot \Theta_{1}$ is monomorphic to a direct product $L_{0} \times L_{1}, L_{\nu} \in \mathcal{O}_{\Theta_{\nu}}$. Moreover, letting $\mathfrak{S}$ denote

$\left({ }^{3}\right)$ For an equivalent formulation of the related problem whether $\mathfrak{T}_{l}$ is monomorphic to $2^{\omega}$, see $\$ 4$, Problem 4 .

$\left({ }^{4}\right)$ The following concepts can be traced through the original paper, [6], or one may consult $[5]$ and $[2$, Chapters 6,8$]$. 
the set of all SI lattices $L$ with universe $|L| \subseteq \omega$, we will obtain an isomorphic representation of $\mathfrak{I}_{l}$ as a lattice of subsets of $\mathcal{S}$ if we pair $\Theta \in \mathbf{T}_{l}$ with the set $\delta-0 \Theta$.

But the most striking facts observed in [6] arise from Lemma 1.4 when $K$ is a finite set of finite lattices. In this case, the lemma implies that every SI $L \epsilon$ OK belongs to the class HSK. Now, there are only a finite number of nonisomorphic lattices in HSK; hence, for present purposes, it may be replaced by a finite representative subclass $\mathcal{F}$. Then every variety $\mathcal{O} \subseteq \mathcal{O K}$ will be generated by a subset of $\mathcal{F}$. Correspondingly, each theory $\Theta \geq \Theta \bar{K}$ is generated by $\Theta \Theta \cap \mathcal{F}$ (and also by a finite lattice $L$, a direct product of the members of $\mathcal{\Theta} \cap \mathcal{F}$ ). Therefore the interval $[\Theta \mathcal{K}, \Omega]$ in $\mathfrak{T}_{l}$ is finite.

Moreover, for two finite SI lattices $L_{0}$ and $L_{1}$, we have

$$
\boldsymbol{\Theta} L_{0}=\boldsymbol{\Theta} L_{1} \leftrightarrow L_{0} \cong L_{1} \text {. }
$$

For, by the above, if they generate the same variety then each is the epimorphic image of a sublattice of the other.

The future application of any of the above summarized results of [6] will be marked by invoking "Jónsson's lemma."

By Jónsson's lemma, the theories of finite lattices, when taken together, form a filter in the lattice of theories. We may ask whether this filter has an alternative definition, using only notions which apply to $\mathfrak{T}_{l}$ as an abstract lattice. An affirmative solution of the following problem would yield such a definition.

Problem 2. Assuming that $\Theta \in \mathbf{T}_{l}$ and $[\Theta, \Omega]$ is finite, must $\Theta$ have the form $\Theta L, L$ a finite lattice?

Alan Kostinsky noticed that this problem is directly equivalent (since $\mathfrak{T}_{l}$ is distributive) to another, now listed as Problem $2^{\circ}$, in $\$ 8$.

2. An equational base for $N_{5}$. Consider the two following equations:

$$
\begin{aligned}
& \eta_{1} \cdot x \cdot(y+u) \cdot(y+v) \leq x \cdot(y+u v)+x u+x v, \\
& \eta_{2} \cdot x \cdot(y+u \cdot(x+v))=x \cdot(y+u x)+x \cdot(x y+u v) .
\end{aligned}
$$

Theorem 2.1. (See Diagram 2.) $N_{5}=\Theta_{l}\left[\eta_{1}, \eta_{2}\right]$.

Rather than verify directly that $\eta_{1}$ and $\eta_{2}$ hold in $N_{5}$, let us observe, more generally, that $\eta_{1}$ and $\eta_{2}$ are valid in every lattice which fails to contain three mutually incomparable elements.

Indeed, assume that $L$ is such a lattice. By writing $a$ ) ( $b$ we signify that $a$ and $b$ are incomparable elements in $L$, i.e. $a\lfloor b$ and $b\lfloor a$. Now, let $a, b, c$, $d \epsilon|L|$. By substituting $a, b, c$ and $d$, respectively, for $x, y, u$ and $v$ in $\eta_{\mu}(\mu=$ $1,2)$, the left and right components of these equations are assigned values $\mathrm{LS}_{\mu}$, $\mathrm{RS}_{\mu}$, respectively, in $L$. The indicated relations between these elements reduce to the fact that $\mathrm{LS}_{\mu} \leq \mathrm{RS}_{\mu}(\mu=1,2)$, for $\mathrm{RS}_{2} \leq \mathrm{LS}_{2}$ clearly is a consequence of the lattice axioms. 
The relation $\mathrm{LS}_{1} \leq \mathrm{RS}_{1}$ is immediate if any two of $b, c, d$ are comparable. For example, $c \leq d$ implies RS $1 \geq a \cdot(b+c d)=a \cdot(b+c) \geq \mathrm{LS}_{1} ; b \leq c$ implies $\mathrm{LS}_{1}=a c \cdot(b+d) \leq \mathrm{RS}_{1} ; c \leq b$ implies $\mathrm{LS}_{1}=a b \leq \mathrm{RS}_{1}$; etc. Hence $\mathrm{LS}_{1} \leq \mathrm{RS}_{1}$.

Similarly, to prove that $\mathrm{LS}_{2} \leq \mathrm{RS}_{2}$ in $L$, we can assume that $\left.a\right)$ (c, that $b$ ) (c and that $a\lfloor b$. Since $a, b, c$ are not all mutually incomparable, we must have $b<a$. Then the cases $a \leq d, d \leq a$ or $c \leq d$ are easily handled. Excluding these cases and recalling that $a$ )(c, we must have $d<c$ (otherwise $a, c, d$ are mutually incomparable). With all the above assumptions, we easily see that the comparability of any two of $a \cdot(b+c), c \cdot(a+d), b+d$ implies that $\mathrm{LS}_{2} \leq \mathrm{RS}_{2}$; whence $\mathrm{LS}_{2} \leq \mathrm{RS}_{2}$.

Corollary 2.2. Assume that in the lattice L every three elements include two comparable elements. Then $L$ can be embedded in a direct power of $N_{5}$.

Proof. From the preceding argument and Theorem 2.1, $L \in \mathcal{O} N_{5}$. It is trivial that every member of HS $N_{5}$ can be embedded in $N_{5}$. Hence, by Jónsson's lemma, $L$ is isomorphic to a subdirect product of sublattices of $N_{5}$.

For the remainder of this section, set $\Theta=\boldsymbol{\Theta}_{l}\left[\eta_{1}, \eta_{2}\right]$. We shall state two lemmas, derive from them Theorem 2.1 , and then prove the lemmas.

[A remark on notation: A term constructed from the elements of a set $F$ using only - will be called the product of $F$ if all elements of $F$ are used in the construction; and will be denoted by $\Pi F$. In order for a set of terms to have a product it must be finite and nonempty. In that case, any two products of $F$, say $\pi_{0}$ and $\pi_{1}$, are equivalent: $\pi_{0} \sim_{\Lambda} \pi_{1}$. Dually, we have the sum of $F$, denoted by $\Sigma F$.

Definition 2.1. A special term of type one (ST1) is any term which has the form $\rho \cdot(\sigma+\tau)$, where each of $\rho, \sigma, \tau$ is a finite product of variables. The dual of an ST1 is an ST2. A special inclusion is any equation $v \leq \phi$, where $v$ is an ST 1 and $\phi$ is an ST2.

Lemma 2.1. For each special inclusion, $\epsilon$, either $\epsilon \in \Lambda$ or $\boldsymbol{\Theta}_{l}[\epsilon]$ contains the modular law: $x \cdot(x y+z) \leq y+(x+y) \cdot z$.

Lemma 2.2. For each term $\sigma$, there are finite, nonempty sets of terms, $F_{1}$ and $F_{2}$, satisfying:

(i) $F_{1}$ consists of ST1's $v$ satisfying $v \leq_{\Lambda} \sigma$; moreover, the equation $\sigma=$ $\sum F_{1} \in \Theta$.

(ii) $F_{2}$ consists of ST2's $\phi$ satisfying $\sigma \leq_{\Lambda} \phi$; moreover, $\sigma=\Pi F_{2} \in \Theta$.

Proof of Theorem 2.1. Earlier on we observed that $\Theta \subseteq \Theta N_{5}$. Conversely, let $\rho=\sigma$ be an arbitrary equation valid in $N_{5}$. Applying Lemma 2.2, we choose a set $F_{1}$ of ST1's such that $\rho \sim_{\theta} \Sigma F_{1}$ and a set $F_{2}$ of ST2's such that $\sigma \sim_{\theta} \Pi F_{2}$. We claim that $\Sigma F_{1} \leq_{\Lambda} \Pi F_{2}$. To see this, choose an arbitrary pair 
$v \in F_{1}, \phi \in F_{2}$. Since $\Theta \subseteq \Theta N_{5}$, the special inclusion $v \leq \phi$ holds in $N_{5}$. Therefore $v \leq \phi \in \Lambda$ by Lemma 2.1, since the modular law fails in $N_{5}$. Now we have

$$
\rho \sim_{\theta} \sum_{F_{1} \leq{ }_{\Lambda}} \prod_{F_{2}} \sim_{\theta} \sigma
$$

and so $\rho \leq_{\theta} \sigma$. The same argument gives $\sigma \leq_{\theta} \rho$. We conclude that $\rho=\sigma \in \Theta$.

Proof of Lemma 2.1. Let $\epsilon$ be any special inclusion

$$
\rho \cdot(\sigma+\tau) \leq \phi+\chi \cdot \psi
$$

which fails to be provable (in $\Lambda$ ). For each term $\pi$, we denote by $\pi^{0}$ the set of all variables appearing in $\pi$. Because each of $\rho, \sigma, \tau$ is a product of variables, and $\phi, \chi, \psi$ are sums of variables, provable inclusions between the se terms depend only upon the variable sets: e.g. $\rho \leq \phi \in \Lambda$ iff $\rho^{0} \cap \phi^{0} \neq 0$.

Since $\epsilon \notin \Lambda$, we have immediately $\rho^{0} \cap \phi^{0}=0$; also either $\rho^{0} \cap \chi^{0}=0$ or $\rho^{0} \cap \psi^{0}=0$, and either $\phi^{0} \cap \sigma^{0}=0$ or $\phi^{0} \cap \tau^{0}=0$. We may as well assume

$$
\left(\phi^{0} \cup \psi^{0}\right) \cap \rho^{0}=0, \quad \phi^{0} \cap \tau^{0}=0 .
$$

Now, let $u$ and $v$ be distinct variables not occurring in $\epsilon$. Suppose $\psi^{0} \cap \tau^{0}=$ 0 . Then $\left(\rho^{0} \cup \tau^{0}\right) \cap\left(\phi^{0} \cup \psi^{0}\right)=0$ by (1). Replacing all variables of $\rho^{0} \cup \tau^{0}$ in $\epsilon$ by $u$, and all those of $\phi^{0} \cup \psi^{0}$ by $v$, we obtain an inclusion which, together with $\Lambda$, yields the inclusion $u \leq v$, i.e. $\boldsymbol{\Theta}_{l}[\epsilon]=\Omega$. Certainly the modular law is derivable from $\epsilon$ in this case. So we may assume

$$
\psi^{0} \cap \tau^{0} \neq 0 \text {. }
$$

(i) $\psi^{0} \cap \sigma^{0}=0 \vee \chi^{0} \cap \rho^{0}=0$;

(ii) $\tau^{0} \cap \chi^{0}=0 \vee \sigma^{0} \cap \phi^{0}=0$;

(iii) $\sigma^{0} \cap \chi^{0}=0 \vee \sigma^{0} \cap \psi^{0}=0 \vee \tau^{0} \cap \chi^{0}=0$.

For the negation of any one of these statements, together with (2), puts $\epsilon \in \Lambda$.

The negation of any of the following statements, together with (1), gives $\boldsymbol{\Theta}_{l}[\epsilon]=\Omega$. Therefore to complete the proof, we assume their validity.

$$
\begin{aligned}
& \text { (i) } \rho^{0} \cap \chi^{0} \neq 0 \vee \tau^{0} \cap \chi^{0} \neq 0 ; \\
& \text { (ii) } \phi^{0} \cap \sigma^{0} \neq 0 \vee \psi^{0} \cap \sigma^{0} \neq 0 ; \\
& \text { (iii) } \phi^{0} \cap \sigma^{0} \neq 0 \vee \chi^{0} \cap \sigma^{0} \neq 0 \vee \chi^{0} \cap \rho^{0} \neq 0 .
\end{aligned}
$$

For example, if (4iii) fails, then $\left(\phi^{0} \cup \chi^{0}\right) \cap\left(\rho^{0} \cup \sigma^{0}\right)=0$. Replacing the variables of $\phi^{0} \cup \chi^{0}$ in $\epsilon$ by $v$ and those of $\rho^{0} \cup \sigma^{0}$ by $u$, we derive $u \leq v$.

It is easy to check that conditions (3) and (4) imply

$$
\psi^{0} \cap \sigma^{0}=0=\tau^{0} \cap \chi^{0}, \quad \phi^{0} \cap \sigma^{0} \neq 0 \neq \rho^{0} \cap \chi^{0} .
$$

By (1), the three nonempty sets $\psi^{0} \cap \tau^{0}, \phi^{0} \cap \sigma^{0}, \rho^{0} \cap \chi^{0}$ are pairwise disjoint. 
To conclude, choose three distinct variables, $x, y, z$. By (1), (2) and (5), we can derive from $\epsilon$ another inclusion $\pi \leq v$ by means of a substitution described as follows: $\rho^{0} \cap \chi^{0} m x, \phi^{0} \cap \sigma^{0} m y, \psi^{0} \cap \tau^{0} m z$, all variables of $\left(\psi^{0}-\tau^{0}\right) \cup$ $\left(\phi^{0}-\sigma^{0}\right)$ are replaced by $y \cdot z$, all variables of $\left(\rho^{0}-\chi^{0}\right) \cup\left(\tau^{0}-\psi^{0}\right)$ are replaced by $x+z$ and all the remaining variables are replaced by $y$. It is easy to see, using (1), (2) and (5), that $x \cdot(x y+z) \leq_{\Lambda} \pi$ and $v \leq_{\Lambda} y+(x+y) \cdot z \cdot$

This concludes the proof. It is only slightly more difficult to show that $\epsilon$ is equivalent to either the modular law, the distributive law $(x \cdot(y+z) \leq y+x \cdot z)$, or the equation $x=y$. This was done independently by Jónsson's student, Dang Xuan Hong.

Proof of Lemma 2.2. Let us first derive from $\eta_{1}$ and $\eta_{2}$ some additional equations of $\Theta, \eta_{3}-\eta_{9}$ below.

The symbolic statements following each of those equations, when properly decoded, form the sketch of a proof that the given equation belongs to $\Theta$. For illustration, suppose the equation in question is $\sigma=\tau$. If $\tau \leq \sigma \in \Lambda$, we will not mention this fact (it will be obvious, if true), but proceed to show that $\sigma \leq \tau \in \Theta$. If the first line of the proof is, for example, "LS $\leq_{\theta} \mathrm{RS}+\sigma_{1}+\sigma_{2}\left(\eta_{1}\right)$," this indicates that $\sigma \leq \sigma_{0}+\sigma_{1}+\sigma_{2}$ is directly derivable from $\eta_{1}$ and the lattice axioms, where $\sigma_{0}$ is some term for which $\sigma_{0} \leq \tau \in \Lambda$. In the following lines, $\sigma_{1}$ and $\sigma_{2}$ will be reduced in the same way, to get eventually $\sigma_{1} \leq_{\theta} \tau, \sigma_{2} \leq_{\theta} \tau$. If, however, the inclusion $\sigma_{2} \leq \tau$ can be obtained by a permutation of the variables in $\sigma_{1} \leq \tau$, then we shall only work to show $\sigma_{1} \leq_{\theta} \tau$ in the remaining lines.

$$
\begin{aligned}
& \eta_{3} \cdot x \cdot(y+z)=x \cdot(x y+z)+x \cdot(y+x z) . \\
& \mathrm{LS} \sim_{\mathbf{\Lambda}} x \cdot[y+z \cdot(x+z)] \text {, } \\
& \sim_{\theta} \text { RS }\left(\eta_{2}\right) \text {. } \\
& \eta_{4} \cdot x+y z=[x+(x+y) \cdot z] \cdot[x+y \cdot(x+z)] . \\
& \mathrm{RS} \leq_{\theta} \mathrm{LS}+z \cdot[x+y \cdot(x+z)]+y \cdot[x+(x+y) \cdot z] \quad\left(\eta_{1}\right), \\
& z \cdot[x+y \cdot(x+z)] \leq_{\boldsymbol{\theta}} \text { LS }\left(\eta_{2}\right) . \\
& \eta_{5} \cdot x \cdot(x u+x v+y)=x \cdot(x u+y)+x \cdot(x v+y) . \\
& \mathrm{LS} \sim_{\mathbf{\Lambda}} x \cdot[x u+(x v+y) \cdot(x+y)], \\
& \sim_{\theta} x \cdot[x u+x \cdot(x v+y)]+x \cdot(x u+y) \quad\left(\eta_{2}\right), \\
& \sim_{\Lambda} \text { RS. } \\
& \eta_{6} \cdot x y+u v=(x+u v) \cdot(y+u v) \cdot(u+x y) \cdot(v+x y) \text {. }
\end{aligned}
$$




$$
\begin{aligned}
& \mathrm{RS} \leq_{\theta} \mathrm{LS}+(x+u v) \cdot(y+u v) \cdot u \cdot(v+x y) \\
&+(x+u v) \cdot(y+u v) \cdot v \cdot(u+x y) \quad\left(\eta_{1}\right), \\
&(x+u v) \cdot(y+u v) \cdot u \cdot(v+x y) \\
& \leq_{\theta} \mathrm{LS}+x u \cdot(y+u v) \cdot(v+x y)+y u \cdot(x+u v) \cdot(v+x y) \quad\left(\eta_{1}\right), \\
& x u \cdot(y+u v) \cdot(v+x y) \leq_{\theta} \mathrm{LS}+x u \cdot(y+x u v) \cdot(v+x y) \quad\left(\eta_{3}\right), \\
& \leq_{\theta} \mathrm{LS}+x u \cdot(y+x u v) \cdot(v+x u y) \quad\left(\eta_{3}\right), \\
& \leq_{\theta} \mathrm{LS}+x u \cdot(x u v+x u y+y v) \quad\left(\eta_{1}\right), \\
& \leq_{\theta} \mathrm{LS} \quad\left(\eta_{5}\right) \cdot \\
& \eta_{7} \cdot x+(y+u v) \cdot w=(x+y+u v) \cdot[x+(y+u) \cdot w] \cdot[x+(y+v) \cdot w] . \mathrm{RS} \leq_{\theta} \rho+\sigma+\tau \quad\left(\eta_{1}\right),
\end{aligned}
$$

where

$$
\begin{aligned}
\rho & =(x+y+u v) \cdot[x+(y+u) \cdot(y+v) \cdot w], \\
\sigma & =(x+y+u v) \cdot(y+u) \cdot w \cdot[x+(y+v) \cdot w], \\
\tau & =(x+y+u v) \cdot(y+v) \cdot w \cdot[x+(y+u) \cdot w] ; \\
\sigma+\tau & \leq_{\theta} \rho \quad\left(\eta_{1}\right)
\end{aligned}
$$

hence we need only show that $\rho \leq_{\theta}$ LS.

$$
\begin{aligned}
& \rho \leq_{\theta} L S+(y+u v) \cdot[x+(y+u)(y+v) \cdot w] \\
& +(y+u) \cdot(y+v) \cdot w \cdot(x+y+u v) \quad\left(\eta_{1}\right), \\
& (y+u v) \cdot[x+(y+u)(y+v) \cdot w] \leq_{\theta} L S+(y+u v) \cdot[x+(y+u) \cdot v w] \quad\left(\eta_{2}\right) \text {, } \\
& \leq_{\theta} \text { LS }\left(\eta_{2}\right) \text {, } \\
& (y+u) \cdot(y+v) \cdot w \cdot(x+y+u v) \\
& \leq_{\theta} \mathrm{LS}+u w \cdot(y+v) \cdot(x+y+u v)+v w \cdot(y+u) \cdot(x+y+u v) \quad\left(\eta_{1}\right), \\
& u w \cdot(y+v) \cdot(x+y+u v) \leq_{\theta} L S+u w \cdot(y+u v+x v) \quad\left(\eta_{1}\right) \text {, } \\
& \leq_{\theta} \operatorname{LS}\left(\eta_{3}\right) \text {. } \\
& \eta_{8}\left(=\eta_{1}{ }^{d}\right) \cdot[x+y \cdot(u+v)] \cdot(x+u) \cdot(x+v) \leq x+y u+y v . \\
& \mathrm{RS} \sim_{\theta} x+y \cdot(u+y v) \cdot(v+y u) \quad\left(\eta_{6}\right), \\
& \geq_{\theta} \text { LS } \cdot[x+y \cdot(u+y v)] \quad\left(\eta_{7}\right) \text {, } \\
& \geq_{\theta} \operatorname{LS}\left(\eta_{7}\right) \text {. } \\
& \eta_{9}\left(=\eta_{2}{ }^{d}\right) \cdot x+y \cdot(u+x v)=[x+y \cdot(u+x)] \cdot[x+(x+y) \cdot(u+v)] \text {. }
\end{aligned}
$$




$$
\begin{aligned}
\mathrm{LS} & \sim_{\theta}[x+y \cdot(u+x)] \cdot[x+y \cdot(u+v)]\left(\eta_{7}\right), \\
& \sim_{\theta}[x+y \cdot(u+x)] \cdot[x+(x+y) \cdot(u+v)] \cdot[x+y \cdot(x+u+v)]\left(\eta_{4}\right), \\
& \sim_{\mathbf{A}} \text { RS. }
\end{aligned}
$$

Having derived $\eta_{3}-\eta_{9}$, we can quickly conclude the proof of Lemma 2.2. Because $\eta_{8}$ and $\eta_{9}$ belong to $\Theta$, we have $\Theta=\Theta^{d}$; and it is only necessary to prove one-half of the lemma. We will prove by induction on the complexity of terms that every term $\sigma$ is $\Theta$-equivalent to a product of ST2's $\phi$ such that $\phi \geq_{\Lambda} \sigma$. $F(G)$, with or without subscripts, will denote a finite, nonempty set of ST2's (ST2's and terms of the type $\rho \cdot \sigma$, where $\rho$ and $\sigma$ are sums of variables).

First note that, given $F$, we can find $G$ with $\Pi F \leq_{\Lambda} \Pi G$ and $x+\Pi F \sim_{\boldsymbol{\theta}}$ $\Pi\{x+\phi: \phi \in G\}$. Indeed, setting $y=u v$ in $\eta_{7}$,

$$
\left.x+u v w \sim_{\boldsymbol{\theta}}(x+u v) \cdot(x+u w) \cdot(x+v u)\right) .
$$

Hence,

$$
x+\prod_{F \sim_{\theta}} \prod\left\{x+\phi_{0} \cdot \phi_{1}: \phi_{0}, \phi_{1} \in F\right\}
$$

And by several applications of $\eta_{7} ;$ if $\phi_{\mu}=\rho_{\mu}+\sigma_{\mu 0} \cdot \sigma_{\mu 1}$, then

$$
x+\phi_{0} \cdot \phi_{1} \sim_{\theta}\left(x+\phi_{0}\right)\left(x+\phi_{1}\right) \cdot \prod_{\kappa, \lambda=0,1}\left[x+\left(\rho_{0}+\sigma_{0 \kappa}\right)\left(\rho_{1}+\sigma_{1 \lambda}\right)\right] .
$$

Now the only nontrivial part of the inductive argument is the passage over + : assume that $\sigma_{\nu} \sim_{\boldsymbol{\theta}} \Pi F_{\nu}$ and $\sigma_{\nu} \leq_{\Lambda} \Pi F_{\nu}(\nu=0,1)$; to similarly reduce $\sigma_{0}+\sigma_{1}$. By the above,

$$
\begin{aligned}
\sigma_{0}+\sigma_{1} \sim_{\boldsymbol{\theta}} & \prod\left\{\prod_{\left.F_{0}+\phi: \phi \in G_{1}\right\}}\right. \\
\sim_{\boldsymbol{\theta}} & \prod\left\{\chi+\phi: \phi \in G_{1}, \chi \in G_{0}\right\},
\end{aligned}
$$

where each term $\chi+\phi \geq_{\Lambda} \sigma_{0}+\sigma_{1}$. Thus it only remains to reduce these very simple terms $\chi+\phi$. That can safely be left to the reader (use $\eta_{6}$, and then $\eta_{7}$ as above). The proof is concluded.

Corollary 2.3. An inclusion $\sigma \leq \tau$ is valid in $N_{5}$ iff every special inclusion $v \leq \phi$, for which $v \leq_{\Lambda} \sigma$ and $\tau \leq_{\Lambda} \phi$, is provable in $\Lambda$.

Remarks. An effective, general method of finding a finite base for a finite lattice is contained implicitly in $[9, \S 3]$.

Each of the sets $\left\{\eta_{1}, \eta_{2}\right\},\left\{\eta_{4}, \eta_{6}, \eta_{7}\right\}$ is an independent base of $\boldsymbol{\theta}_{5}$. The independence may be deduced semantically from various lattices in our diagrams. For this theory, and also $8 M_{3}$ (Diagram 1 and [7]), the minimum number of variables appearing in a base is four.

It seems remarkable that a theory can be defined as in Corollary 2.3. A 
similar statement, obtained by replacing $N_{5}$ by $M_{3}$ and $\Lambda$ by the theory of modular lattices, will be proved by Comer and Hong [3]. The mystery of these results may be partly dissipated by what follows, for instance Remark 5.2 and Example 7.1. Whether they have an interesting common generalization is as yet unclear.

3. Splittings of $\mathfrak{T}_{l}$. A splitting pair $\left({ }^{5}\right)\left(\right.$ in $\mathfrak{T}_{l}$ ) is an ordered pair of theories, $\langle\mathrm{H}, \Theta\rangle$, such that every theory $\mathrm{K} \in \mathrm{T}_{l}$, satisfies precisely one of the conditions $\mathrm{H} \leq \mathrm{K}, \mathrm{K} \leq \Theta$. Such a pair "splits" the lattice $\mathfrak{T}_{l}$ into two disjoint intervals, $[H, \Omega]$ and $[\Lambda, \Theta]$.

Lemma 3.1. Assume that $\left\langle\mathrm{H}_{\nu}, \Theta_{\nu}\right\rangle(\nu=0,1)$ are splitting pairs. Exactly one of the following conditions is true:

(i) $\left\langle\mathrm{H}_{0}, \Theta_{0}\right\rangle=\left\langle\mathrm{H}_{1}, \Theta_{1}\right\rangle$;

(ii) $\mathrm{H}_{0} \leq \Theta_{1}$ and $\mathrm{H}_{1} \leq \Theta_{0}$;

(iii) $\mathrm{H}_{0}+\Theta_{0} \leq \Theta_{1}$ and $\mathrm{H}_{0} \leq \mathrm{H}_{1} \cdot \Theta_{1}$;

(iv) $\mathrm{H}_{1}+\Theta_{1} \leq \Theta_{0}$ and $\mathrm{H}_{1} \leq \mathrm{H}_{0} \cdot \Theta_{0}$.

A most accessible example is the pair $\langle\Omega, \Delta\rangle$. Another is the pair $\left\langle\mathrm{M}, \boldsymbol{\Theta}_{N_{5}}\right\rangle$, where $M$ is the theory based on the modular law of Lemma 2.1. That this is indeed a splitting pair follows from the well-known fact that an arbitrary lattice is nonmodular iff it contains $N_{5}$ isomorphically. There are denumerably many splitting pairs in $\mathfrak{T}_{l}$ (as will be shown by Example 5.2.)

The splitting pairs in $\mathfrak{T}_{l}$ evidently constitute a bi-unique function. The domain and range of this function fit into a classification of irreducible theories which we shall now discuss, deferring a deeper analysis of the splittings of $\mathfrak{T}_{l}$ until $\$ 5$.

4. Irreducible theories. Given a lattice $L$, a join-irreducible $(J I)$ in $L$ is any element $a \neq 0$ for which $a=b+c$ always implies either $a=b$ or $a=c$. A JI element of the dual lattice of $L$ is said to be meet-irreducible (MI) in $L$. One speaks also of join-prime elements $(a \leq b+c \Rightarrow a \leq b \vee a \leq c)$ and meet-prime elements $(a \geq b \cdot c \Rightarrow a \geq b \vee a \geq c)$. In case $L$ is complete, it is customary to define four stronger properties, using, in place of + and $\cdot$, the operations of complete join and complete meet. To be specific, a strictly join-irreducible (SJI) element, a, satisfies by definition: $a=\bigvee X \Rightarrow a \in X$; and a strictly join-prime (SJP) element has the characteristic property: $a \leq \bigvee X \Rightarrow(\exists x \in X)(a \leq x)$. A strictly meet-irreducible $(S M I)$, or strictly meet-prime $(S M P)$, element satisfies the respective dual restriction.

[Remark 4.1. With reference to $\S_{3}$, observe that the splitting pairs $\langle a, b\rangle$ in a complete lattice (e.g. in $\mathfrak{T}_{l}$ ) map the SJP's bi-uniquely onto the SMP's. Given $a$,

$\left({ }^{5}\right)$ We depart slightly from the terminology of Philip Whitman [14]. 
we must have $b=\bigvee(|L|-[a, 1])$, and dually $a=\bigwedge(|L|-[0, b])$. $]$

When we apply these definitions to $\mathfrak{T}_{l}$, several give equivalent properties of theories. In fact, Theorem 4.2 below implies that all four categories of join-irreducible theory coalesce into one class, JI. And, of course, meet-irreducible and meet-prime-theories are the same because $\mathfrak{T}_{l}$ is a distributive lattice. Altogether, however, five distinct classes of meet-irreducible theories require our attention. Besides MI, SMI and SMP, as defined above, they are: the set SI, consisting of all theories $\Theta$ where $L$ is SI; and the set FSI, consisting of all theories $\boldsymbol{\Theta} L$, where $L$ is finite and $\mathrm{SI}$.

Lemma 4.1 [6, Lemma 4.3]. $\bigwedge$ FSI $=\Lambda$.

Theorem 4.2. Each join-irreducible theory is strictly join-prime (and conversely). An equivalent condition on $\Theta$ is that $\nabla_{l} \Theta=\{1\}(\$ 1)$.

Proof. Assume that $\Theta \in J$ I. Then $\Theta \neq \Lambda$. By Lemma 4.1, choose a finite lattice $L$ satisfying $\Theta L \unrhd \Theta$. By Jónsson's lemma, there are only finitely many theories $\Theta L+\boldsymbol{\Theta}_{l}[\epsilon], \epsilon \in \Theta$. If they are $\Theta L+\boldsymbol{\Theta}_{l}\left[\epsilon_{\nu}\right](\nu \leq \cdot k)$, then clearly

i.e.

$$
\Theta \leq \boldsymbol{\Theta} L+\boldsymbol{\Theta}_{l}\left[\epsilon_{0}, \cdots, \epsilon_{K}\right]
$$

$$
\Theta=\Theta \cdot \Theta L+\underset{\nu \leq K}{\bigvee} \boldsymbol{\Theta}_{l}\left[\epsilon_{\nu}\right]
$$

Since $\Theta \cdot \Theta L<\Theta$ and $\Theta$ is join-irreducible, it is generated by one of the equations $\epsilon_{\nu}$, so $1 \in \nabla_{l} \Theta$. Again, we cannot have $\Theta=\Theta_{l}\left[\gamma_{0}, \gamma_{1}\right]$ unless $\Theta=\Theta_{l}\left[\gamma_{0}\right]$ or $\Theta=$ $\boldsymbol{\Theta}_{l}\left[\gamma_{1}\right]$. Thus $2 \notin \nabla_{l} \Theta$. Hence by Lemma 1.2, $\nabla_{l} \Theta=\{1\}$. Moreover, $\Theta$ is compact and join-irreducible in the distributive lattice $\mathfrak{S}_{l}$, and therefore strictly joinprime in $\mathfrak{T}_{l}$.

On the other hand, if $\nabla_{l} \Theta=\{1\}$ and $\Theta=\Theta_{0}+\Theta_{1}$, then $\Theta$ has a finite base $\Sigma \subseteq \Theta_{0} \cup \Theta_{1}$. By possibly discarding some equations of $\Sigma$ we obtain an independent base $\Sigma^{\prime}$ for $\Theta$ which, by hypothesis, consists of only one equation $\epsilon$, say $\epsilon \in \Theta_{0}$. Then $\Theta=\Theta_{0}$.

\section{Theorem 4.3. SMP $\subset$ FSI $\subset$ SMI $\subset$ SI $\subset$ MI.}

Proof. (1) SI $\subset$ MI. The inclusion merely reformulates a consequence of Jónsson's lemma that was proved by him and restated in our $\$ 1$. Example 4.2 below presents a meet-irreducible theory which has no subdirectly irreducible generating lattice.

(2) SMI $\subset$ SI. Assume that $\Theta$ is strictly meet-irreducible. $\Theta \Theta$ is generated by its subdirectly irreducible members, i.e. $\Theta=\wedge([\Theta, \Omega] \cap \mathrm{SI})$; and so $\Theta \in \mathrm{SI}$. But if $L$ is an infinite SI lattice which is the directed union of finite lattices, for instance the lattice $M_{\infty}$ of [7], then $\Theta L$ is the proper intersection of the theories 
of these finite lattices (by Jónsson's lemma); and so $\boldsymbol{\Theta} L \in \mathrm{SI}-\mathrm{SMI}$ in this case. $\Lambda$ is another example. By Lemma 4.1, $₫ \notin$ SMI. However, $\Lambda$ is the theory of the (SI) lattice of all partitions of $\omega .\left({ }^{6}\right)$ Jónsson had proved that $\Lambda \in$ MI by another method [6, Theorem 4.6].

(3) FSI $\subset$ SMI. Let $L$ be a finite SI lattice. Then the interval $[\boldsymbol{\theta} L, \Omega]$ is finite and, by (1), $\Theta L$ is meet-irreducible. Therefore, clearly $\Theta L \in$ SMI. Pass to Example 4.1 below for the proof that inclusion (3) is proper.

(4) SMP $\subset$ FSI. Assume that $\Theta$ is strictly meet-prime. Then, by Lemma 4.1, there is a $\Theta^{\prime} \in \mathbf{F S I}$ for which $\Theta^{\prime} \leq \Theta$. By Jónsson's lemma, $\Theta=\boldsymbol{\Theta} L$ with $L$ finite. Since $\Theta$ is meet-prime, it must be generated by one of the SI images of $L$. Therefore $\Theta \in$ FSI. For a theory in FSI - SMP, we refer to Example 5.1 below, where it is shown that $M_{3}$ is not strictly meet-prime. This concludes the proof of Theorem 4.3 .

In $\$ 5$ and $\$ 6$, we will attack the problem of which finite SI lattices $L$ satisfy $\boldsymbol{Q} L \in \mathrm{SMP}$; and we shall find a very satisfactory solution, indeed an effective method for deciding whether $L$ belongs to this class, and when the answer is affirmative, for constructing an equation $\epsilon$ such that $\left\langle\boldsymbol{\Theta}_{l}[\epsilon], \boldsymbol{\Theta} L\right\rangle$ splits $\mathfrak{T}_{l}$.

An analogous, and apparently more difficult, problem is the following.

Problem 3. Find necessary and sufficient algebraic conditions for a SI lattice to possess a SMI theory.

By Example 5.2 and Theorem 4.3, we shall have

$$
\#(\text { SMP })=\#(\text { FSI })=\boldsymbol{\kappa}_{0},
$$

and it is not difficult to modify the method of [9] to show that

$$
\exists(\mathbf{S I})=\#(\mathbf{M I})=2^{\boldsymbol{N}_{0}} \text {. }
$$

The cardinal $\kappa=$ (SMI) lies somewhere in between and has not been determined. It would be of some theoretical interest to do so because $\kappa$ is also the least cardinal $\alpha$ for which $\mathfrak{T}_{l}$, can be embedded in the set lattice $2^{\alpha}$. This fact, which applies, more generally, to every complete, distributive and algebraic $\left({ }^{7}\right)$ lattice, is probably well known. The argument is simple: From [2, Chapter 8 , Theorem 16], every theory $\Theta$ is a meet of strictly meet-irreducible theories. Therefore, quite trivially, the map $\Theta m \rightarrow$ SMI $-[\Theta, \Omega]$ embeds $\mathfrak{T}_{l}$ into the lattice of subsets of SMI. But conversely, if $F$ embeds $\mathfrak{F}_{l}$ into $2^{X}$, then we can inject SMI into $X$ by selecting, for each $\Theta \in \mathrm{SMI}$, a point $x \in F(\bar{\Theta})-F(\Theta)$, where $\bar{\Theta}$ is the smallest theory strictly extending $\Theta$.

$\left({ }^{6}\right)$ There is a well-known theorem due to Whitman [Bull. Amer. Math. Soc. 52 (1946), $507-522]$, stating that every countable lattice can be embedded in this partition lattice. O. Ore [Duke Math. J. 9 (1942), 626] proved that the lattice is simple (a fortiori, subdirectly irreducible).

$\left({ }^{7}\right)$ A lattice in which every element is the join of compact elements. 
Problem 4. How many SMI theories are there?

Two examples close this section.

Example 4.1. There are several known examples ([1] and [16]) of (modular) lattice varieties $\mathcal{O}$ which are not generated by any set of finite lattices. For such a $\mathcal{O}, 0 \bigcirc$ is not a meet of theories of finite lattices. However, it is the meet of strictly meet-irreducible theories (Birkhoff, op. cit.), and consequently the set SMI - FSI must be nonempty. A concrete example of a theory in this set is $L_{r}$, where $L_{r}$ is the lattice of subspaces of a three-dimensional vector space over the rational numbers. The proof is beyond our means here and we must refer to [1], in particular his remark in $\$ 2$, from which it can be constructed with a little effort.

Example 4.2. The infinite lattice $R$ of Diagram 13 satisfies $\Theta R \in \mathbf{M I}$ - SI. The proof (only sketched here) illustrates very nicely the power of Jónsson's lemma. Let us first show that $R$ is meet-irreducible. For each $\nu<\omega$, the elements 0 , $1, a_{\nu}$ and all the elements above or to the left of $a_{\nu}$ in Diagram 13 constitute a sublattice of $R$ which we will call $R_{\nu}$. Each lattice $R_{\nu}, \nu>0$, is subdirectly irreducible, in fact every proper homomorphism of $R_{\nu}$ identifies $a_{\nu}$ and $b_{\nu}$. In addition, $R$ is the union of the chain $\left\{R_{\nu}\right\}$, and so $\Theta R=\bigwedge\left\{\Theta R_{\nu}: \nu>0\right\}$, the intersection of a chain of meet-irreducible theories (Theorem 4.3). This fact alone directly implies $\Theta R \in \mathbf{M I}$.

It requires a bit more effort to see that $\Theta R \notin S I$. Suppose, to the contrary, that $B$ is subdirectly irreducible and $\Theta B=\Theta$. By Jónsson's lemma, we have a homomorphism $F$ from $C$ onto $B$, where $C$ is monomorphic to an ultrapower of $R$. But $R$ is a special sort of lattice, whose ultrapowers are easily visualized.

Whereas $R$ itself is composed of $R_{0}$ and $\omega$ copies of $N_{5}$, sandwiched between 0 and 1 and connected one to the next as shown in Diagram 13; every ultrapower of $R$ is isomorphic to some lattice $D$ obtained from $R$ by adding, between 0 and 1 , a set of additional components, each new component consisting of countably many copies of $N_{5}$, strung out like the integers but connected one to the next as in $R$.

Thus we may assume that $C$ is a sublattice of one such lattice $D$. If we agree that the elements of $R$ other than 0 and 1 also form a component of $D$, then the product and sum of two elements from distinct components of $D$ will be equal, respectively, to 0 and 1 .

Next, we apply, once again, the initial assumption $B=B R$. Setting $\nu>0$, we have $R_{\nu} \in \mathcal{O} B$, and hence $B$ contains a subset order-isomorphic to $R_{\nu}$. (By Jónsson's lemma, $R_{\nu} \in \mathbf{H S P}_{u} B$, and Baker [1] observed that this implies that every finite partly ordered set embedded in $R_{\nu}$ can also be embedded in B.) From this we obtain a subset of $C$ which is order-isomorphic to $R_{\nu}$ and on which the function $F$ is one-to-one. Now the crucial fact is that $D$ contains only one subset order-isomorphic to $R_{\nu}$, namely $\left|R_{\nu}\right|$. (Induct on $\nu$. The anomalous element $d_{0}^{\prime}$ was placed just for this purpose.) Hence, letting $\nu$ tend to infinity gives: $|R| \subseteq|C|$ and $F$ is one-to-one on $|R|$. 
To conclude, by showing that $B$ is not subdirectly irreducible we will contradict the original assumption. For each $\nu \geq 0$, the congruence relation $S_{\nu}$ over $D$, generated by collapsing the quotient $\left[a_{\nu}, b_{\nu}\right]$ in $R$, also collapses $\left[e_{\mu}, d_{\mu}\right]$ and $\left[a_{\mu}, c_{\mu}\right]$ for $\mu>\nu$, but nothing more. Since $F$ is a monomorphism when restricted to $R$,

$$
\overline{\mathrm{S}}_{\nu}=\left\{\langle F x, F y\rangle: x, y \in|C| \& S_{\nu} x y\right\}
$$

is a nontrivial congruence of $B$. But the intersection of all of the $\bar{S}_{\nu}$ is the equality relation of $B$. Hence $B$ is not subdirectly irreducible.

\section{Bounded homomorphisms.}

Definition 5.1. An equation $\epsilon$ and a finite, SI lattice $L$ will be called conjugates of one another when every theory $\mathrm{K} \in \mathrm{T}_{l}$ satisfies precisely one of the conditions $\epsilon \epsilon \mathrm{K}, L \in \mathcal{O}$; or more concisely, if $\left\langle\boldsymbol{\Theta}_{l}[\epsilon], \boldsymbol{\Theta}\right\rangle$ is a splitting pair. A finite, SI lattice $L$, which has a conjugate equation (i.e. $\Theta L \in \mathrm{SMP}$ ), will be called a splitting lattice. And an equation $\epsilon$ which possesses a conjugate lattice (i.e. $\left.\boldsymbol{\Theta}_{l}[\epsilon] \in \mathbf{J I}\right)$ will be termed irreducible.

Observe now, that, on the basis of $\$ 4$, every splitting pair in $\mathfrak{T}_{l}$ is associated with a pair of conjugates, $\langle\epsilon, L\rangle$, from which it can be obtained in the manner described above. Observe also that, given a conjugate pair $\langle\epsilon, L\rangle$, although every conjugate of $\epsilon$ is isomorphic to $L$ (Jónsson's lemma), the conjugates of $L$ are just those equations $\epsilon^{\prime}$ which are equivalent to $\epsilon$ in lattice theory.

With the aid of the concept of bounded homomorphism, we can draw a rather pretty picture of these notions, the principal features of which will be two theorems below, 5.1 and 5.4 .

Definition 5.2. A lattice homomorphism $g: A \rightarrow B$ will be called upper bound$e d$ if every nonempty set of the form $\{x \in|A|: g(x) \leq b\}, b \in|B|$, has a largest element. $g: A \rightarrow B$ is lower bounded iff $g: A^{d} \rightarrow B^{d}$, between the dual lattices, is upper bounded. Finally, we call a homomorphism bounded if it is both upper and lower bounded.

Note that, if $A$ is finite, then every homomorphism from $A$ is bounded; but, in general, if $B \subseteq C$ and $g: A \rightarrow B$ is bounded, it does not follow that $g: A \rightarrow C$ is bounded.

Theorem 5.1. Eacb of the following conditions is necessary and sufficient for a finite, SI lattice $B$ to be a splitting lattice:

(i) $B$ is a bounded epimorphic image of a free lattice (which is necessarily finitely generated).

(ii) Every lattice homomorphism $g: A \rightarrow B$, where $A$ is finitely generated, is bounded.

This surprising characterization is a fountain rich in corollaries. It has first 
to be primed with a proof, and that will occupy the greater part of this section. For now, observe only that the theorem appears to present us a feasible program for testing whether a given finite lattice $L$ is a splitting lattice.

In outline, we would first decide whether $L$ is subdirectly irreducible, and that is very easy. Next, we would "construct" some homomorphism collapsing a finitely generated free lattice onto $L$, and then attempt to decide whether the homomorphism is bounded, in order at last to apply Theorem 5.1. In $\$ 6$, we shall display an effective and mechanical procedure for performing the test along these lines.

A word about free lattices before we tackle Theorem 5.1. A lattice $F$ is free if it has a generating set $Y \neq 0$ such that every mapping of $Y$ into another lattice $A$ can be extended to map $F$ homomorphically into $A$. Under this assumption, $Y$ is said to freely generate $F$, and we write $F=F L(Y)$. The nature of free lattices was clarified by Phillip Whitman [12]. A lattice $F$ generated by a set $Y \neq 0$ is freely generated by $Y$ iff $Y$ is a set of mutually incomparable elements and whenever $a_{0}, a_{1}, b_{0}, b_{1} \in|F|$ and $y \in Y$, we have

(W1) If $a_{0} \cdot a_{1} \leq y \leq b_{0}+b_{1}$, then for some $\mu, \nu \in\{0,1\}, a_{\mu} \leq y \leq b_{\nu}$.

(W2) If $a_{0} \cdot a_{1} \leq b_{0}+b_{1}$, then either $a_{0} \leq b_{0}+b_{1}$ or $a_{1} \leq b_{0}+b_{1}$ or $a_{0} \cdot a_{1} \leq$ $b_{0}$, or $a_{0} \cdot a_{1} \leq b_{1}$.

We need, also, the more general notion of a $\Theta$-free lattice $F=\mathrm{FL}(\Theta, Y)(\Theta \epsilon$ $\mathrm{T}_{l}$ ). Here, one assumes that $F \in \mathcal{O} \Theta$ and that every map from $Y$ into a member of $\mathcal{O} \Theta$ can be extended. With every theory $\Theta$ and nonvoid set $Y$, there exists, up to an isomorphism which fixes $Y$, exactly one $\Theta$-free lattice $F L(\Theta, Y)$. Of course, the structure of these lattices is usually far less transparent than that of the $\Lambda$ free lattices $\mathrm{FL}(Y)$.

The following discussion is much smoother if we have some particular free lattices available for instant reference. Throughout $\$ \S 5,6$ and $7, F L(\omega)$ will denote a fixed lattice freely generated by the denumerable set $\omega$, and for $1 \leq \kappa<\omega$, $\mathrm{FL}(\kappa)$ will denote the sublattice of $\mathrm{FL}(\omega)$, (freely) generated by $\kappa$. The elements of $\mathrm{FL}(\omega)$ will be called words, and when it is necessary to name a specific word, we will use the symbols $\mathbf{a}, \mathbf{b}, \mathbf{c}$, etc. for distinct free generators (letters of $\mathrm{FL}(\omega)$ ). Finally, we will make use of the obvious mapping $\sigma \rightsquigarrow \sigma^{b}$, which converts terms of the basic equational language into words of $F L(\omega)$ by matching the variables of the language with the elements of $\omega$ in natural order. To illustrate, for $\sigma=x+y \cdot z, \sigma^{b}=\mathbf{a}+\mathbf{b} \cdot \mathbf{c}$. Of course, for an arbitrary equation $\epsilon=\sigma=\tau$, we have $\epsilon \epsilon \Lambda$ iff $\sigma^{b}=\tau^{j}$. And if $\epsilon$ involves at most the first $\kappa$ variables, and $L$ is any lattice, then $\epsilon \in L \Leftrightarrow f\left(\sigma^{b}\right)=f\left(\tau^{b}\right)$ whenever $f: \mathrm{FL}(\kappa) \rightarrow L$. Also under this assumption, if $\Theta \in \mathbf{T}_{l}$ and $b$ is the canonical map of $\mathrm{FL}(\kappa)$ onto a $\mathrm{FL}(\Theta, \kappa)$, then $\epsilon \epsilon$ $\Theta \Leftrightarrow b\left(\sigma^{b}\right)=b\left(\tau^{b}\right)$.

Proof of Theorem 5.1. The proof will be divided into two parts. 
I. Assume that $B$ is finite, subdirectly irreducible, and satisfies 5.1(i). To find a conjugate equation for $B$.

Take any bounded homomorphism $f: \mathrm{FL}(\kappa) \rightarrow B$, where $1 \leq \kappa<\omega$, and let $u$, $v \in|B|$ be such that $u<v$ ( $v$ covers $u$ ) and every proper homomorphism of $B$ identifies $u$ with $v$ ( $u, v$ exist because $B$ is SI and finite). Because $f$ is bounded and onto, the set $f^{-1}\{u\}$ has a largest element, $w_{0}$, and $f^{-1}\{v\}$ has a smallest element, $w_{1}$.

Now let $\pi$ be any term representing $w_{0}$, i.e. $\pi^{b}=w_{0}$; similarly, let $\rho^{b}=w_{1}$. Denote by $\epsilon$ the equation $\rho \leq \pi$. I claim that $\epsilon$ is a conjugate irreducible equation of the lattice $B$. Certainly $\epsilon \notin \Theta B$, because $f\left(\pi^{b}\right)=u$ while $f\left(\rho^{b}\right)=v$. But if $\Theta$ is a theory not contained in $\Theta B$, then, directly from Lemma 5.1 below, $\Theta$ contains an inclusion $\sigma \leq \tau$ in which $f\left(\sigma^{b}\right)=v$ and $f\left(\tau^{b}\right)=u$. By construction, $w_{1} \leq \sigma^{b}$ and $\tau^{b} \leq w_{0}$ in $\mathrm{FL}(\kappa)$, or what is equivalent, $\rho \leq_{\Lambda} \sigma$ and $\tau \leq_{\Lambda} \pi$. And this last, combined with $\sigma \leq_{\theta} \tau$, puts $\epsilon \in \Theta$.

We require five secondary lemmas for the proof of Theorem 5.1. The second and the fourth of these seem independently interesting.

Lemma 5.1. Assume that $1 \leq \kappa \leq \omega$ and that $f: \mathrm{FL}(\kappa) \rightarrow B$ is an epimorphism. Assume also that the lattice $B$ bas an atomic quotient $[u, v]$ (i.e. $v$ covers $u$ ) col. lapsed by every proper homomorphism of $B$. Then an equivalent condition for a theory $\Theta \in \mathbf{T}_{\text {l }}$ to satisfy $\Theta \nless \Theta B$ is that $\Theta$ contains some inclusion $\sigma \leq \tau$ where $f\left(\sigma^{b}\right)=v$ and $f\left(\tau^{b}\right)=u$.

Proof. Under the assumptions, each of these inclusions certainly fails in $B$. In the other direction, suppose that $\Theta \not{\Theta B}$. Let $R$ be the congruence relation of $\mathrm{FL}(\kappa)$ induced by the map $f$, and let $R(\Theta)$ be the congruence induced by the canonical map $\mathrm{FL}(\kappa) \rightarrow \mathrm{FL}(\Theta, \kappa)$. Now, the congruence $R^{\prime}=R+R(\Theta)$ is larger than $R$, otherwise $B$ would be an epimorphic image of $F L(\Theta, \kappa)$ and we should have $\Theta \leq \Theta B$. Therefore, the obvious map $B \rightarrow \mathrm{FL}(\kappa) / R^{\prime}$ is not monomorphic, and must collapse $[u, v]$.

Consequently, choosing words $w_{0}<w$ such that $f\left(w_{0}\right)=u$ and $f(w)=v$, we will have $w_{0} R^{\prime} w$, and $w_{0}$ can be connected to $w$ by some finite sequence $w_{0}$, $w_{1}, \cdots, w_{2 \mu}=w$ satisfying $w_{2 \nu} R w_{2 \nu_{+1}}$ and $w_{2 \nu_{+1}} R(\boldsymbol{\theta}) w_{2 \nu_{+}}$(for $<\mu$ ). We can even require that $w_{0} \leq w_{\lambda} \leq w$ for $0 \leq \lambda \leq 2 \mu$, whence we get $f\left(w_{\lambda}\right) \in\{u, v\}$ for $\lambda \leq 2 \mu$, because $u<v$ in $B$. Now, we can choose a $\lambda<2 \mu$ for which $f\left(w_{\lambda}\right)=u$ and $f\left(w_{\lambda+1}\right)=$ $v$. Clearly $w_{\lambda} R(\Theta) w_{\lambda+1}$ must hold. Therefore, picking $\sigma$ and $\tau$ to satisfy $\sigma^{b}=$ $w_{\lambda+1}, \tau^{b}=w_{\lambda}$, we will have $\sigma \leq \tau \in \Theta\left(\tau^{b} R(\Theta) \sigma^{b}\right.$ is equivalent to $\left.\tau=\sigma \in \Theta\right)$, $f\left(\sigma^{b}\right)=v$ and $f\left(r^{b}\right)=u$. This is the desired result.

Lemma 5.2. Assume that $g: A \rightarrow F$ where $A$ is finitely generated and $F$ is a free lattice. Then $g$ is bounded. $\left({ }^{8}\right)$

$\left.{ }^{8}\right)$ This lemma overlaps recent results of Grätzer and Lakser concerning "covers" in free lattices. 
Proof. It will suffice to show that $g$ is upper bounded. A dual argument gives the lower bounds.

Let $F=\mathrm{FL}(Y)$ and let $G$ be a finite (nonvoid) generating set for $A$. Call an element, $w$, of $F$ "good" if $g^{-1}(\{x \in F: x \leq w\})$ is either empty, or has a greatest element. We use Whitman's description of the ordering in $F((W 1)$ and (W2) above) to show that the good elements of $F$ form a sublattice including $Y$, thereby completing the proof.

Step 1. Let $w \in Y$ and assume $g^{-1}(\leq w) \neq 0$. Let $a=\bigvee\left[G \cap g^{-1}(\leq w)\right]$. Clearly $g(a) \leq w$. Induct on $x \in|A|$ to show $g(x) \leq w \Rightarrow x \leq a$. If $x \in G$, it is trivial. If it is true for $x$ and $y$, it is surely true for $x+y$; and if $g(x \cdot y)=g(x)$. $g(y) \leq w$, then $g(x) \leq w$ or $g(y) \leq w$ (by (W1)), so $x \leq a$ or $y \leq a-$ a fortiori $x \cdot y \leq a$.

Step 2. Let $w=w_{0}+w_{1}$ where $w_{0}, w_{1}$ are good. Then the sum

$$
a=\bigvee\left[\left(G \cap g^{-1}(\leq w)\right) \cup g^{-1}\left(\leq w_{0}\right) \cup g^{-1}\left(\leq w_{1}\right)\right]
$$

certainly exists in $A$, and if $g^{-1}(\leq w)$ is nonempty then $a$ is its largest element. To see this, just induct on $x$ as before to show $g(x) \leq w \Rightarrow x \leq a$, using now (W2) in place of (W1).

Step 3. Let $w=w_{0} \cdot w_{1}$ where $w_{0}, w_{1}$ are good. If $g^{-1}(\leq w) \neq 0$, then the largest element of the set is obviously the product of the corresponding elements for $w_{0}$ and $w_{1}$. Hence $w$ is good.

For the next two lemmas, let $(\mathrm{P})$ denote any one of the three properties of homomorphisms given by Definition 5.2. Lemma 5.3 is obvious.

Lemma 5.3. Assume that $f: B \rightarrow C$ and $g: A \rightarrow B$ are lattice bomomorphisms. If $f$ and $g$ are (P) bomomorphisms (in particular, if $B$ is finite and $g$ is $(\mathrm{P})$ ), then so is $f g$. If $f g$ is $(\mathrm{P})$ and $g$ is epimorphic ( $f$ is monomorphic), then $f$ (respectively $g$ ) is also a $(\mathrm{P})$ bomomorphism.

Lemma 5.4. Assuming that the lattice $B$ is finitely generated, the following conditions are equivalent:

(i) There exists a (P) bomomorphism collapsing a free lattice onto $B$.

(ii) Every lattice bomomorphism $g: A \rightarrow B$, where $A$ is finitely generated, is a (P) bomomorphism.

Proof. That (ii) implies (i) is obvious. Conversely, assume that $f: F \rightarrow B$ is a (P) homomorphism, with $F$ free, and let $g: A \rightarrow B$ where $A$ is generated by $\kappa$ elements, $1 \leq \kappa<\omega$. Choose some $b: \mathrm{FL}(\kappa) \rightarrow A$. Since $f$ is epimorphic and $\mathrm{FL}(\kappa)$ is free, there is an $b_{1}: \mathrm{FL}(\kappa) \rightarrow F$ such that $f b_{1}=g b$. By the two preceding lemmas, $g h=f b_{1}$ is (P). Hence, again by Lemma 5.3 ( $b$ is epimorphic), $g$ is (P). The proof is complete.

Definition 5.3. Given $1 \leq \kappa<\omega$ and $\lambda<\omega$, the lattice $C(\kappa, \lambda)$ is constructed by the following procedure. As the universe of $C(\kappa, \lambda)$, we take the finite subset 
of $\mathrm{FL}(\kappa)$ consisting of the largest element, together with all the words $\sigma^{\prime} \epsilon$ $|\mathrm{FL}(\kappa)|$ where the term $\sigma$ contains at most $\lambda$ occurrences of,$+ \cdot$, , and all the words which are finite products of these. The sum and product in $C(\kappa, \lambda)$ are defined in the only possible way to make the lattice ordering the same as in $F L(\kappa)$. Thus products are the same as in $\mathrm{FL}(\kappa)$, but many sums are increased.

$D(\kappa, \lambda)$ is constructed by a dual treatment. Its universe consists of the smallest word in $\mathrm{FL}(\kappa)$, all words in $\mathrm{FL}(\kappa)$ having "complexity" $\leq \lambda$, and all the sums of these words.

The utility of these lattices in this context arises from the following lemma, part of which is well known and implies Lemma 4.1.

Lemma 5.5. Given $\kappa$ and $\lambda$, every equation $\epsilon \notin \Lambda$ baving complexity at most $\lambda$ and containing at most $\kappa$ variables, fails in $C(\kappa, \lambda)$ and in $D(\kappa, \lambda)$. Moreover, $C(\kappa, \lambda)$ is an upper bounded epimorpbic image of $\mathrm{FL}(\kappa)$, while $D(\kappa, \lambda)$ is a lower bounded epimorph of $\mathrm{FL}(\kappa)$.

All assertions of this lemma follow readily from a consideration of the morphisms $f: \mathrm{FL}(\kappa) \rightarrow C(\kappa, \lambda)$ and $g: \mathrm{FL}(\kappa) \rightarrow D(\kappa, \lambda)$ which map the generators of $\mathrm{FL}(\kappa)$ identically. If $w \in|C(\kappa, \lambda)|$, then $f(w)=w$ and, for $w^{\prime} \in|\mathrm{FL}(\kappa)|, f\left(w^{\prime}\right) \leq w$ iff $w^{\prime} \leq w$. Dual considerations hold for $g$.

Proof of Theorem 5.1. II. By Lemma 5.4, conditions 5.1(i) and 5.1(ii) are equivalent for every finite lattice $B$. Hence, in view of part $I$ of the proof, it only remains to demonstrate 5.1 (i) under the assumption that $B$ is a splitting lattice. We now make that assumption. $\epsilon$ denotes a conjugate equation of $B$.

Take, by Lemma 5.5, $\kappa, \lambda$ so large that $\epsilon$ fails in $C(\kappa, \lambda)$ and $D(\kappa, \lambda)$. By the defining property of splitting pairs, we must have $\Theta C(\kappa, \lambda)+\Theta D(\kappa, \lambda) \leq \Theta B$. Then by Jónsson's lemma, applied to these three finite lattices ( $B$ is $\mathrm{SI}$ ), each of $C(\kappa, \lambda), D(\kappa, \lambda)$ has a sublattice which collapses homomorphically onto $B$. From this fact, with several applications of all the Lemmas 5.3, 5.4 and 5.5, one can obtain that $B$ is the upper bounded epimorph of a free lattice, and also the lower bounded epimorph of a free lattice. This is decisive; it gives, immediately, 5.1(i) via Lemma 5.4. The straightforward details of these last steps are left to the reader. Our proof of Theorem 5.1 is now complete.

The remainder of the section carries some easy corollaries of Theorem 5.1 and its proof, results concerning the splittings of $\mathfrak{T}_{l}$, free lattices and their finite, bounded homomorphic images, which should help to clarify the significance of these related concepts. The most important of these, perhaps more interesting than Theorem 5.1, are Theorems 5.4 and 5.5.

All these results may be thought to have a rather special flavor because the kernel of reference, the class of splitting lattices, is admittedly somewhat restricted. Yet we feel that the connections and applications demonstrated below and in $\$ \$ 6-8$ argue otherwise. Many concrete examples will be developed in $\$ 7$. 
This whole theory depends heavily on Jónsson's lemma and appears to have no analogue in the equational model theory of well-known algebraic varieties other than lattices, for example the variety of groups.

Our first observations concern the class $\mathcal{B}$ which consists of the finite lattices which are bounded epimorphic images of free lattices. By Theorem 5.1, the splitting lattices are the SI members of $\mathfrak{B}$. By Lemma 5.2, among the members of $B$ we find every finite sublattice of $\operatorname{FL}(\omega)$. (However, see Example 7.10 below.)

Now, a very simple argument, based on Lemmas 5.3 and 5.4 , shows that $\mathcal{B}$ is closed under the formation of sublattices, homomorphic images, and direct products with finitely many factors. Therefore, by Jónsson's lemma, $\{\Theta L: L \in \mathbb{B}\}$ is a subfilter in $\mathfrak{T}_{l}$ of the filter comprised of the theories of finite lattices $(\$ 1$, Problem 2); and we have the following useful complement of Theorem 4.3:

Corollary 5.2. If $B$ is a splitting lattice, or more generally if $B \in \mathfrak{B}$, and if $A$ is a SI lattice belonging to $\bigcirc_{B}$, then $A$ is a splitting lattice. In the symbolism of $\$ 4$, if $\mathrm{H} \in \mathbf{S M P}, \Theta \in \mathbf{M I}$ and $\mathrm{H} \leq \Theta$, then also $\Theta \in \mathbf{S M P}$.

The free lattices possess one very strict structural property that is obviously preserved by bounded epimorphisms and is therefore shared by the lattices of $\mathfrak{B}$.

Corollary 5.3. If $B$ is a splitting lattice, or more generally if $B \in \mathcal{B}$, and if $u, x, y, z \in|B|$, then

(i) $u=x \cdot y=x \cdot z$ implies $u=x \cdot(y+z)$;

(ii) $u=x+y=x+z$ implies $u=x+y \cdot z$.

Proof. Assume, for instance, that $f: F \rightarrow B$ is bounded, that $F$ is free and that $x \cdot y=x \cdot z$ in $B$. Let $w_{0}, w_{1}$ and $w_{2}$ be the largest elements of $F$ mapping onto $x, y$ and $z$, respectively. Then $w_{0} \cdot w_{1}$ and $w_{0} \cdot w_{2}$ are the largest elements mapping onto $x \cdot y$ and $x \cdot z$. Whence $w_{0} \cdot w_{1}=w_{0} \cdot w_{2}$. Hence by $[8, \mathbb{9} 1]$, $w_{0} \cdot w_{1}=w_{0} \cdot\left(w_{1}+w_{2}\right)$. Applying $f$, we get $x \cdot y=x \cdot(y+z)$.

Example 5.1. From this corollary, $M_{3}$ (Diagram 1) is [subdirectly irreducible but] not a splitting lattice; the same applies to the lattices of Diagrams 3, 4 and 10. Thus the intersection of all theories $\Theta \not \Theta \Theta M_{3}$ is itself a subtheory of $\Theta M_{3}$. We do not know whether the intersection is $\Lambda$. (It is, if the answer to Problem 6 below is yes.)

Example 5.2. It is a well known fact that $\mathrm{FL}(\omega)$ contains a copy of $N_{5}$. For its universe we can take the words $\mathbf{c}, \mathbf{a c}, \mathbf{a b}+\mathbf{c}, \mathbf{a b}+\mathbf{a c}$ and $\mathbf{a}(\mathbf{a b}+\mathbf{c})$. By a result of Jonsson, the quotient $[\mathbf{a b}+\mathbf{a c}, \mathbf{a} \cdot(\mathbf{a b}+\mathbf{c})]$ contains a copy of $\mathrm{FL}(\omega)$, hence also a copy of $N_{5}$; therefore a copy of $N_{6}$ (Diagram 11) can be found in $\mathrm{FL}(\omega)$. Iteration of this argument serves to embed all the SI lattices $N_{\mu}$ (Diagram 11 ) into $\mathrm{FL}(\omega)$. So here we have an infinite family of splitting lattices descended from $N_{5}$.

Problem 5. Are conditions 5.3(i) and 5.3(ii) of the corollary sufficiently strong 
to characterize $B$ ? Or what is equivalent, does every finite, SI lattice fulfilling the se conditions possess a conjugate equation?

Remark 5.1. It was conjectured in [8] that all finite lattices isomorphic to a sublattice of $\mathrm{FL}(\omega)$ can be characterized by three conditions $\left({ }^{9}\right)$ : 5.3(i), 5.3(ii) and Whitman's (W2) (p. 18 of this section). In view of Theorem 6.3 of the following section, this conjecture would be proved by an affirmative solution of our Problem 5.

Remark 5.2. We do not need this, and will not prove it, but it seems noteworthy that a finite lattice belongs to $B$ iff, for some $\kappa, \lambda$, it belongs to both ${ } C(\kappa, \lambda)$ and $O_{D}(\kappa, \lambda)$ (Definition 5.3). In fact, if $B \in \mathfrak{B}$, then $B$ is a homomorph of both lattices when $\kappa$ and $\lambda$ are sufficiently large.

It hardly seems remarkable that irreducible equations (Definition 5.1) should be closely related to the atomic quotients in free lattices. In fact, the two notions are, in a sense, equivalent, and that is the topic of the next group of observations. Our interest here is naturally confined to the $\mathrm{FL}(\kappa), \kappa<\omega$, since the covering relation in $\mathrm{FL}(\omega)$ is trivially empty.

Let us remark that, outside of a few comments in Whitman's papers, very little has been published on the covering relation in free lattices, and so, in view of what follows, most of our results concerning the splittings of $\mathfrak{T}_{l}$ (irreducible equations, splitting lattices) should add to the knowledge of that subject.

First we return to the proof of Theorem 5.1, part I. There it was shown that if $f$ is a bounded map of $\mathrm{FL}(\kappa), 1 \leq \kappa<\omega$, onto a finite, SI lattice $B$, then a conjugate equation for $B$ of the form $\rho \leq \pi$ is constructed by choosing any atomic quotient $[u, v]$ which witnesses the subdirect irreducibility of $B$ and taking $\pi, \rho$ to satisfy: $\pi^{b}$ is the largest word in $F L(\kappa)$ representing $u$ by $f ; \rho^{b}$ is the smallest representing $v$. The equivalent conjugate equations $\rho \cdot \pi=\rho$ and $\pi=\pi+\rho$ may then each be obtained by "equating the endpoints of an atomic quotient of FL $(\kappa)$." Indeed, for example, any word $w \in|\mathrm{FL}(\kappa)|$ which satisfies $\rho^{\prime} \cdot \pi^{b} \leq$ $\omega \leq \rho^{b}$ will be equal to either $\rho^{b} \cdot \pi^{b}$ or $\rho^{b}$ depending on whether $f(w)=u$ (and $\left.w \leq \pi^{b}\right)$ or $f(w)=v$ (and $w \geq \rho^{b}$ ); and so $[\rho \cdot \pi]^{b}<\rho^{b}$ in $\operatorname{FL}(\kappa)$ (but not in $\mathrm{FL}(\kappa+1)$, of course).

To expand on this, recall that with any atomic quotient $[x, y]$ of an arbitrary lattice $L, L$ possesses a largest congruence relation separating $x$ and $y$. Still keeping the assumptions of the last paragraph, it now becomes obvious that the kernel of the map $f$ must be the largest congruence over $\mathrm{FL}(\kappa)$ which separates $\rho^{b} \cdot \pi^{b}$ from $\rho^{b}$.

Now we may ask what happens in the other direction. We take $w_{0}=\tau^{b}$ and $w_{1}=v^{\prime}$ such that $w_{0}<w_{1}$ in $\operatorname{FL}(\kappa)(\kappa<\omega$ is fixed), and we let $R$ denote the

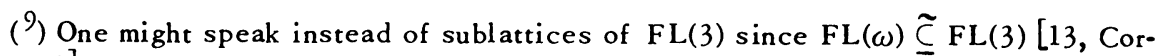
ollary 3.1 . 
largest congruence of $\mathrm{FL}(\kappa)$ which fails to collapse $\left[w_{0}, w_{1}\right]$. For $\lambda$ large, the projections onto $C(\kappa, \lambda)$ and $D(\kappa, \lambda)$ separate $w_{0}$ and $w_{1}$, by the proof sketched for Lemma 5.5. The projection of $\mathrm{FL}(\kappa)$ onto $B=\mathrm{FL}(\kappa) / R$ therefore factors through both maps and so, in view of Lemmas 5.3 and 5.5 , it is bounded. $B$ is clearly finite and SI-by all rights (and Theorem 5.1) a splitting lattice. Finally, $\tau=v$ and $B$ are conjugates. In fact, $\tau=v \notin \Theta$ iff the kernel $R(\Theta)$ which maps $\mathrm{FL}(\kappa) \rightarrow \mathrm{FL}(\Theta, \kappa)$ satisfies $R(\Theta) \subseteq R$ (definition of $R$ ) and this implies $B \in \Theta_{\Theta}$ $(B \in \mathrm{HS} F \mathbf{F L}(\Theta, \kappa))$.

To summarize the last three paragraphs:

Theorem 5.4. Let $f: \mathrm{FL}(\kappa) \rightarrow B, \kappa<\omega$. Then $B$ is a splitting lattice iff there exist two terms $\tau, v$ in the first $\kappa$ variables sucb that

(i) $\tau^{b}<v^{b}$ in $\mathrm{FL}(\kappa)$; and

(ii) $\operatorname{Ker}(f)$ is identical with the largest congruence of $\mathrm{FL}(\kappa)$ that separates $\tau^{b}$ from $v^{j}$.

Moreover, in this case, $r=v$ is a conjugate equation of $B$.

Theorem 5.5. (Compare Theorem 4.2 and Definition 5.1.) Let $\epsilon$ be any equation. $\epsilon$ is irreducible iff $\Theta_{l}[\epsilon]=\Theta_{l}[\tau=v]$, where $\tau^{b}<v^{b}$ in some $\operatorname{FL}(\kappa)$.

One further remark introducing a problem: If $\left[\sigma^{b}, \rho^{b}\right]$ contains an atomic quotient $\left[\tau^{b}, v^{b}\right]$ in $\operatorname{FL}(\kappa)$, then $\tau=v$ is derivable from $\sigma=\rho$, and the latter equation fails in the splitting lattice conjugate to the former. Conversely, if $\sigma \leq_{\Lambda} \rho$ and $\sigma=\rho$ fails in a splitting lattice $B$, there will be a bounded homomorphism $b$ : $\mathrm{FL}(\kappa) \rightarrow B(\kappa<\omega)$ where $\sigma^{b}, \rho^{b} \in|\mathrm{FL}(\kappa)|$ and $b\left(\sigma^{b}\right)<b\left(\rho^{b}\right)$. Choosing $u$ and $v$ so as to have $b\left(\sigma^{b}\right) \leq u<v \leq b\left(\rho^{b}\right)$ and taking for $w_{0}$ the smallest word representing $v$ by $b$ and for $w_{1}$ the largest representing $u$, one can show that $\left[\left(w_{0}+\sigma^{b}\right) \cdot w_{1}\right.$, $\left.w_{0}+\sigma^{b}\right]$ is an atomic quotient contained in $\left[\sigma^{b}, \rho^{b}\right]$.

Problem 6. The following statements are equivalent. Are they valid?

(1) $\bigwedge \mathrm{SMP}=\Lambda$.

(2) Every theory $\Theta>\Lambda$ contains an irreducible equation.

(3) Whenever $1 \leq \kappa<\omega$ and $w_{0}<w_{1}$ are words in $\mathrm{FL}(\kappa)$, there are words $w_{2}$ and $w_{3}$ satisfying $w_{0} \leq w_{2}<w_{3} \leq w_{1}$ in $\operatorname{FL}(\kappa)$.

Three little corollaries are yet to come and then we will let the case rest. In the light of Theorem 5.5, the second of these merely restates the first.

Corollary 5.6. Every splitting lattice other than a two-element lattice contains $N_{5}$ as sublattice. Every irreducible equation, other than $x=y$ and its equivalents, belongs to the modular theory $\mathrm{M}$.

Proof. Any subdirectly irreducible lattice with more than two elements contains either $N_{5}$ or $M_{3}$. From Corollary 5.2 and Example 5.1, no splitting lattice contains $M_{3}$. 
Again, if $\epsilon$ is irreducible and $\epsilon \notin M$ then the conjugate lattice of $\epsilon, L$, is modular (i.e. $L \in \mathcal{O M}$ ). Hence if $L$ has more than two elements, $M_{3} \subseteq L$ and we get the same contradiction. So $F|L|=2$ and $\Theta_{l}[\epsilon]=\Omega$.

Corollary 5.7. Any atomic quotient in $\mathrm{FL}(\kappa)$ which collapses in $\mathrm{FL}(\Delta, \kappa)$ collapses also in $\mathrm{FL}(\mathrm{M}, \kappa)$. ( $\Delta$ is distributive theory.)

Corollary 5.8. (See Theorem 8.1 for a related fact.) Assume that $L$ is a finite lattice with more than one element. Then $2 \in \nabla_{l} \Theta L$.

Proof. Otherwise ( $(1$ and Theorem 4.2) $\Theta$ is join-irreducible. By Corollary 5.6, that gives $\Theta L=\Omega$ or $\Theta L \leq M$. But $\Theta L \leq M$ is ruled out by Jónsson's lemma for there are infinitely many theories extending $M[7]$. Hence $\boldsymbol{\theta} L=\Omega$. This implies $\sharp|L|=1$, contradiction.

6. Constructive methods. This section makes a constructive supplement to the theory of splittings developed over the past three sections. It contains a simple algorithm to decide whether a homomorphism is bounded; reveals some connections with well-known decision problems; and hints at possible improvements and generalizations-in particular the algorithm is useful in cases where a finite lattice "splits" some proper interval $[\Theta, \Omega]$ of theories. As a consequence of the basic result (Lemma 6.1) we shall obtain an algorithm for deciding whether a finite lattice is a splitting lattice, and constructing a conjugate equation (Theorem 6.1); an algorithm for testing whether one word covers another in a free lattice (Theorem 6.2); and an algorithm for recognizing whether a finite lattice can be embedded into $\mathrm{FL}(\omega)$ and for constructing an embedding if one exists (Theorem 6.3).

[Remark 6.1. Upon learning of the results that were to go into this section, Bjarni Jónsson kindly lent to the author some unpublished notes concerning sublattices of free lattices. The notion of bounded homomorphism was absent but, in a different setting, some of our results were already present in Jónsson's notes. Namely, the concept of a "limit table" (Definition 6.2) was used in what I call the standard form, and a statement equivalent to Theorem 6.4 was proved. Further, an important result of this section, Theorem 6.3, was suggested by reading these notes and would not have appeared otherwise. The argument for Theorem 6.3, as indicated in the proof, incorporates a crucial idea due to Jónsson. I have adopted Jónsson's perspicuous notation for the table of limits.]

The precise, mathematical use of the term "recursive" to indicate, loosely speaking, the existence of an algorithm-an "effective," "mechanical" method-to determine membership (as applied to sets of equations, or to isomorphism invariant classes of finite algebras, and to various other sets or classes of finitary entities) should be familiar. The problem whether a given lattice theory $\Theta$ is recursive is synonymous with what has been called the word problem for $\mathrm{FL}(\Theta, \omega)$ : An equation $\sigma=\tau$ belongs to $\Theta$ iff $\sigma$ and $\tau$ represent the same element of $\mathrm{FL}(\Theta, \omega)$; and 
we ask for an algorithm whereby one may determine, for every pair of words presented in this way, whether they are equal. Every finite lattice has, of course, a recursive theory. And $\Lambda$ is recursive; we use Whitman's solution of the word problem for $F L(\omega)$ (derived directly from (W1) and (W2), \$5) all the time. But, after these, the next simplest theory, M, already presents a very difficult word problem. (We attained a minor result on this problem with Corollary 5.7: if $\sigma^{b}<\tau^{b}$ in $\mathrm{FL}(\kappa)$, then $\sigma \sim_{M} \tau$ iff $\sigma \sim_{\Delta} \tau$.) At present, it is not known whether $\mathrm{M}$ is recursive (see [15]).

Because of this, we do not really expect that an algorithm for recognizing irreducible equations will fall into our lap; even though we will have an algorithm for the corresponding lattices. Indeed, let $\epsilon$ be any equation. By Lemma 1.1, we can construct immediately a one element base $\left\{\epsilon^{\prime}\right\}$ for $M+\Theta_{l}[\epsilon]$. Then by Corollary 5.7, $\epsilon \in M$ iff $\epsilon^{\prime}$ is irreducible and $\epsilon^{\prime} \epsilon \Delta$. Thus, if we had such an algorithm, we could solve the modular word problem. We do have a weaker result: The irreducible equations are recursively enumerable. By Theorem 5.5, they may be enumerated by first using the algorithm that justifies Theorem 6.2 below, to obtain a list of all equations $\sigma=\rho$ such that $\sigma^{b}<\rho^{b}$ in some $\mathrm{FL}(\kappa)$, and then augmenting the list to include all equivalent equations (relative to $\Lambda$ ).

Definition 6.1. Suppose that $g: A \rightarrow B$, and $x \in|B|$. The upper limit of $x$ (for $g$ ) will be the largest element of $A$ which represents $x$ under $g$, if such an element exists. The lower limit of $x$ will be the least representative of $x$, when it exists. The limits of $x$ will be denoted by $g^{-}(x)$ and $g_{-}(x)$, respectively.

If $g$ is bounded, then clearly $g_{-}$is additive, $g^{-}$is multiplicative, and both are one-to-one.

The central problem that must be solved to get constructive results from Theorems 5.1 and 5.4 is obviously the following: Suppose that we have obtained in some way a complete description (or a diagram) of a finite lattice $B$, together with a selected system of generators of $B$, say $b_{0}, \cdots, b_{\kappa-1}(\kappa<\omega)$. Then the canonical map $f: F L(\kappa) \rightarrow B$ that matches the letters (free generators) of $F L(\kappa)$ in natural order with $b_{0}, \cdots, b_{\kappa-1}$ is completely determined. How do we decide whether $f$ is bounded and find the limits if it is?

Definition 6.2. Given $g: A \rightarrow B$, a limit table for $g$ consists of two systems of functions $\alpha_{\lambda}, \beta_{\lambda}(\lambda<\omega)$ mapping $|B|$ into $|A|$ and satisfying, for every $\lambda<\omega$ and $x \in|B|$,

(i) $g \alpha_{\lambda}(x)=g \beta_{\lambda}(x)=x$;

(ii) $\beta_{\lambda+1}(x) \leq \beta_{\lambda}(x), a_{\lambda}(x) \leq a_{\lambda+1}(x)$;

(iii) whenever $g(y)=x$, there is a $\mu<\omega$ such that $\beta_{\mu}(x) \leq y \leq \alpha_{\mu}(x)$.

If $B$ is finite, a limit table for $g$ will eventually close iff $g$ is bounded; if the limits exist, for some $\lambda$ we will have $\alpha_{\lambda}=g^{-}$and $\beta_{\lambda}=g_{-}$and of course $a_{\mu}=a_{\lambda}$ and $\beta_{\mu}=\beta_{\lambda}$ for all $\mu>\lambda$. In $\S 7$, we will be depicting these tables by rectangular 
arrays. The $x$ th row will be devided into a lower half, containing from left to right the entries $\beta_{0}(x), \beta_{1}(x), \ldots$; and an upper half containing $\alpha_{0}(x), \alpha_{1}(x), \ldots$ The $\lambda$ th column, $\alpha_{\lambda}, \beta_{\lambda}$, may be viewed as the $\lambda$ th simultaneous approximation to the limits of $g$.

Now let us return to the central problem and, for the moment, assume that each element of $B$ oscurs just once in the list $b_{0}, \cdots, b_{\kappa-1}$. Then we define the standard limit table for $B$ in the following manner. For $b \in|B|$, let $S(B, b)$ be the family of all nonempty sets $U \subseteq|B|$ such that $b \leq \bigvee U$, and define $M(B, b)$ dually. Now we put $a_{0}\left(b_{\nu}\right)=\beta_{0}\left(b_{\nu}\right)=\nu$, the letter of $\mathrm{FL}(\kappa)$ representing $b_{\nu}$ under $f$. And for $\lambda>0$, put

$$
\begin{aligned}
& a_{\lambda}(x)=\bigvee_{U \in M(B, x)} \bigwedge_{y \in U} a_{\lambda-1}(y), \\
& \beta_{\lambda}(x)=\bigwedge_{V \in S(B, x)} \bigvee_{z \in V} \beta_{\lambda-1}(z) .
\end{aligned}
$$

In the general case of an arbitrary $f: \mathrm{FL}(\kappa) \rightarrow B$, we construct a special limit table for $f$ on a different pattern. For $b \in|B|$, let $S_{0}(B, b)$ be the family of all sets $U=\{c, d\} \subseteq|B|$ such that $b \leq c+d$ while $b \measuredangle c, b \measuredangle d$, and if $c_{0} \leq c$ and $d_{0} \leq d$ and $b \leq c_{0}+d_{0}$ then $c_{0}=c$ and $d_{0}=d$. Define $M_{0}(B, b)$ dually. For the first column of a special table we choose representative words, $\alpha_{0}(x)$ and $\beta_{0}(x)$ for $x$, so that $\alpha_{0}$ and $\beta_{0}$ are isotonic functions $\left(x \leq y \Leftrightarrow \alpha_{0}(x) \leq \alpha_{0}(y) \Leftrightarrow \beta_{0}(x) \leq\right.$ $\left.\beta_{0}(y)\right)$, and for every letter $\mathbf{a}$ of $\mathrm{FL}(\kappa), \beta_{0} f(\mathbf{a}) \leq \mathbf{a} \leq a_{0} f(\mathbf{a})$. This presents no problem. Then for $\lambda>0$ we put

$$
\alpha_{\lambda}(x)=\alpha_{\lambda-1}(x)+\underset{U \in M_{0}(B, x)}{\bigvee} \bigwedge_{y \in U} a_{\lambda-1}(y)
$$

and the $\beta_{\lambda}$ are defined by a dual process.

We remark that condition 6.2 (iii) defining a table of limits is verified for the standard and the special tables just defined, by inducting on the complexity of the word $y$; and the other conditions are obvious. All the columns in these tables, with the exception of the first column in a standard table, are isotonic functions. In theoretical considerations the standard table is most amenable. But the special tables are the best means available for the actual calculation of limits ( $\$ 7$ ). Both types of tables have the obvious and desirable property, deriving from the uniform manner of passing from one column to the next, of closing with the column $\left\langle a_{\lambda}\right.$, $\left.\beta_{\lambda}\right\rangle=\left\langle f^{-}, f_{-}\right\rangle$just as soon as $\left\langle a_{\lambda}, \beta_{\lambda}\right\rangle=\left\langle a_{\lambda+1}, \beta_{\lambda+1}\right\rangle$; to test whether the table has closed we need only apply Whitman's algorithm (for $\mathrm{FL}(\kappa)$ ) to determine if the last two columns are identical.

At this juncture the basic algorithm, rumored to exist, is almost visible. Given $f: \mathrm{FL}(\kappa) \rightarrow B(\kappa, B$ finite $)$ we begin to construct the columns of a special limit table for $f$, one at a time. If $f$ is bounded, the table will close with a repeated 
column. As soon as this happens, the limits of $f$ will be displayed to satisfaction in the last column. There is a fly in the ointment: If $f$ is not bounded, we are presumably committed to calculate forever. But the fly is a ghost.

Lemma 6.1. Assume that $B$ is a finite lattice of cardinality $\xi$, and that $g$ : $\mathrm{FL}(\kappa) \rightarrow B$ where $1 \leq \kappa<\omega$. Then

(i) If the standard table of limits for $B$ closes, the limits will be attained on or before the $2^{\xi \cdot \xi}$ th column.

(ii) If a special table of limits for $g$ closes, the limits will be attained on or before the $\xi \cdot 2^{\xi \cdot \xi}$ th column.

These numbers bounding the number of steps required to reach a decision by our algorithm are undoubtedly grossly excessive. The lemma is, of course, actually true for each of the independent halves of the tables. We will prove it only for the sequences of upper approximations. If $U$ and $V$ are subsets of a lattice, write $V \ll U$ if for every $u \in U$ there is $v \in V$ with $v \leq u$.

Proof of Lemma 6.1. To prove (i), let $\left\{\alpha_{\lambda}\right\}$ be the upper approximants from the standard limit table for $B$, and assume that eventually $a_{\gamma}=a_{\gamma+1}$. Analogous considerations will hold for the lower approximants.

(P) Suppose that $W$ is a finite nonvoid subset of $F L(\xi)$, that $\lambda \geq 0$, and that $\bigwedge W \leq \alpha_{\lambda}(b)$ where $b \in|B|$. Then one of the following is valid:

(1) $w \leq \alpha_{\lambda}(b)$ for some $w \in W$;

(2) $\lambda>0$ and there is a set $U \in M(B, b)$ satisfying $W \ll\left\{\alpha_{\lambda-1}(y): y \in U\right\}$.

We can prove this by induction on $\lambda$, with $W$ fixed. The case $\lambda=0$ is immediate from the argument for $\lambda=1$, so we begin with $\lambda=1$. Assume that $\wedge W \leq$ $a_{1}(b)$. By Whitman's (W2) (or rather its valid extension to an arbitrary number of terms), either (P1) holds or $\Lambda W$ is dominated by one of the summands of $\alpha_{1}(b)$. Assume the latter, say

$$
\bigwedge W \leq \bigwedge\left\{\alpha_{0}(y): y \in U\right\}
$$

where $U \in M(B, b)$. From the construction of the standard table, every $\alpha_{0}(y)$ is a letter of $\mathrm{FL}(\xi)$; hence by (W1) there is $w_{y} \in W$ such that $w_{y} \leq a_{0}(y)$. Thus (P2) holds with this choice of $U$.

Now suppose that $(\mathrm{P})$ is true of $W$ for $\lambda=\mu-1>0$. Assume that $\Lambda W \leq \alpha_{\mu}(b)$. Again, we use (W2) to reduce to the case

$$
\bigwedge w \leq \bigwedge\left\{\alpha_{\mu-1}(y): y \in U\right\}
$$

where $U \in M(B, b)$. Letting $U^{0}$ be the set of those $y \in U$ for which $W \ll\left\{\alpha_{\mu-1}(y)\right\}$ we choose, with the help of the induction assumption, for each $y \in U-U^{0}$ a set $U_{y} \in M(B, y)$ satisfying $W<<\left\{\alpha_{\mu-2}(z): z \in U_{y}\right\}$. Now, to complete the proof of (P), set

$$
U^{1}=U^{0} \cup \cup\left\{U_{y}: y \in U-U^{0}\right\}
$$


Then clearly $U^{1} \in M(B, b)$ and, since $a_{\mu-2} \leq a_{\mu-1}, W \ll\left\{a_{\mu-1}(z): z \in U^{1}\right\}$.

To get on with the proof of 6.1 (i) let us consider the equivalence relation over $\omega$ defined by: $p \mu \nu$ iff for every $b, b^{\prime} \in|B|$ we have $a_{\mu+1}\left(b^{\prime}\right) \leq a_{\mu}(b) \Leftrightarrow a_{\nu+1}\left(b^{\prime}\right) \leq a_{\nu}(b)$.

(Q) $p \mu \nu$ implies $p \mu+1 \nu+1$.

Indeed, assume that $p \mu \nu$, and that $\alpha_{\mu+2}\left(b^{\prime}\right) \leq a_{\mu+1}(b)$. To get that $a_{\nu+2}\left(b^{\prime}\right) \leq a_{\nu+1}(b)$, we must consider a typical summand of $a_{\nu+2}\left(b^{\prime}\right)$, say $w_{0}=$ $\bigwedge\left\{a_{\nu+1}(y): y \in U\right\}$ where the set $U \in M\left(B, b^{\prime}\right)$. Let $W=\left\{a_{\mu+1}(y): y \in U\right\}$. Since $\alpha_{\mu+2}\left(b^{\prime}\right) \leq \alpha_{\mu+1}(b)$, a fortiori, $\bigwedge W \leq \alpha_{\mu+1}(b)$, and we plug this relation into statement (P). In case (P1) holds we have $\alpha_{\mu+1}(y) \leq \alpha_{\mu+1}(b)$ for a certain $y \in U$. Then $y \leq b$ and hence

$$
w_{0} \leq \alpha_{\nu+1}(y) \leq \alpha_{\nu+1}(b) .
$$

On the other hand, if (P2) holds then we have a set $V \in M(B, b)$ such that $W<<$ $\left\{\alpha_{\mu}(v): v \in V\right\}$. Then for each $v \in V$ there is $y_{\nu} \in U$ satisfying $\alpha_{\mu+1}\left(y_{\nu}\right) \leq \alpha_{\mu}(v)$. These relations imply their twins $\alpha_{\nu+1}\left(y_{\nu}\right) \leq \alpha_{\nu}(\nu)$, since we assumed that $p \mu \nu$. . Whence

$$
\begin{aligned}
w_{0} & \leq \bigwedge\left\{\alpha_{\nu_{+}}\left(y_{v}\right): v \in V\right\} \\
& \leq \bigwedge\left\{a_{\nu}(v): v \in V\right\} \leq \alpha_{\nu_{+1}}(b),
\end{aligned}
$$

since $V \in M(B, b)$. Thus, in every case $w_{0} \leq a_{\nu+1}(b)$; and because $a_{\nu+2}\left(b^{\prime}\right)$ is a sum of such words, we have $\alpha_{\nu+2}\left(b^{\prime}\right) \leq \alpha_{\nu+1}(b)$. The remainder of the proof of (Q) just repeats the above argument.

With (Q) completely proved, 6.1(i) comes very quickly. We can assume that $\xi>1$, and with that assumption a simple combinatorial argument shows that $p$ divides $\omega$ into less than $2^{\xi \cdot \xi}$ classes of integers. We infer the existence of $\mu<$ $\nu<2^{\xi \cdot \xi}$ such that $p \mu \nu$. By (Q), $\mu$ will be $p$-equivalent to arbitrarily large integers; in particular, from the initial assumption, we will have $p \mu \gamma$ where $\alpha_{\gamma}=$ $a_{\gamma+1}$. But then the definition of $p$ requires $\alpha_{\mu+1} \leq \alpha_{\mu}$, and so $\alpha_{\mu+1}=\alpha_{\mu}$.

We have here a much better estimate of how far the standard table must be calculated to reach a decision: one need only carry the upper table far enough to uncover a relation $p \mu \nu$ with $\mu<\nu$; and analogously for the lower table $\left\{\beta_{\lambda}\right\}$.

To prove 6.1(ii) we switch notation and now let $\left\{\alpha_{\lambda}\right\}$ denote the upper approximants in some special table of limits for $g$. Again we assume that eventually $a_{\gamma}=a_{\gamma+1}$. Then $g$ is upper bounded, and by Lemma 5.4 and the proof of 6.1(i), the upper approximants of the standard table will also come to a standstill-at least by the $2^{\xi \cdot \xi}$ th column. Now we consider another sequence of functions $\left\{\alpha_{\lambda}^{0}\right\}$, constructed on exactly the same pattern as the upper approximants in the standard table, but beginning with $\alpha_{0}^{0}=\alpha_{0}$. Because the first entries of the standard table are the letters of $\mathrm{FL}(\xi)$, the upper part of that table translates onto $\left\{\alpha_{\lambda}^{0}\right\}$ in a natural way and we infer that $\alpha_{\mu}^{0}=a_{\nu}^{0}$, where $\mu=2^{\xi \cdot \xi}<\nu$. The proof is concluded 
by an easy induction showing that, in general, $\alpha_{\lambda} \leq \alpha_{\lambda}^{0} \leq \alpha_{\xi \cdot \lambda}$.

Recall from $\S 5$ that $B$ is the class of finite lattices which are bounded epimorphs of free lattices, and that splitting lattices are the SI members of $\mathbb{B}$.

Theorem 6.1. $B$ is recursive.

Proof. Let $B$ be any finite lattice, $\xi=*|B|$ and $\lambda=\xi \cdot 2^{\xi \cdot \xi}$. To decide whether $B \in \mathfrak{B}$, we construct the first $\lambda+1$ columns, $\alpha_{\mu}, \beta_{\mu}(\mu \leq \lambda)$, of a special table of limits for some $g: \mathrm{FL}(\xi) \rightarrow B$. By Lemmas 5.4 and $6.1, B \in B_{\text {iff }} \alpha_{\lambda-1}=$ $\alpha_{\lambda}$ and $\beta_{\lambda-1}=\beta_{\lambda}$.

Now suppose we do have $B \in \mathcal{B}$ and $B$ is SI. Choose $u<v$ in $B$ witnessing the subdirect irreducibility of $B$. Then by the proof of Theorem 5.1, part I, $\rho \leq \pi$ is a conjugate equation of $B$ whenever $\rho^{b}=\beta_{\lambda}(v)$ and $\pi^{b}=\alpha_{\lambda}(u)$. If we merely assume $u<v$ in $B$ (SI not required) then we can only conclude that $\alpha_{\lambda}(u) \cdot \beta_{\lambda}(v)<\beta_{\lambda}(v)$ in $\operatorname{FL}(\xi)$.

Remark 6.2. If $B \notin \Re$, but $u \prec v$ witnesses the subdirect irreducibility of $B$, we can still obtain some useful information by extending a special table indefinitely. We then have a system of equations decreasing in strength- $\epsilon_{\mu}=\rho_{\mu} \leq \pi_{\mu}$ where $\rho_{\mu}^{b}=\beta_{\mu}(v)$ and $\pi_{\mu}^{b}=\alpha_{\mu}(u)_{-w i t h}$ the property that, in general, $B \notin \mathcal{O} \Theta$ iff, for some $\mu, \epsilon_{\mu} \in \Theta$ (by Lemma 5.1). A sequence of equations with these properties will be called a discriminating system for $B$. We may also have occasion to speak of a discriminating system for a theory. The proper definition is obvious.

Theorem 6.2. The relation of covering in free lattices $\mathrm{FL}(\kappa)$ is recursive.

Proof. Suppose that we are given two words, $w_{0}<w_{1}$, of $\mathrm{FL}(\kappa)$, where $1 \leq$ $\kappa<\omega$. To test whether $w_{0}<w_{1}$ in $\mathrm{FL}(\kappa)$, we first find an upper bound $\lambda$ for the complexity of the words. Then the projection $f$ of $F L(\kappa)$ onto the finite lattice $C(\kappa, \lambda)$ of Definition 5.3 separates $w_{0}$ from $w_{1}$; and by Theorem 5.4 we infer that if $w_{0} \prec w_{1}$, then there will be some homomorphism $b: C(\kappa, \lambda) \rightarrow B$ where $B$ is a splitting lattice, satisfying $b f\left(w_{0}\right)<b f\left(w_{1}\right)$.

Next we make a list of the (finitely many) congruences $R$ over $C(\kappa, \lambda)$ which separate $f\left(w_{0}\right)$ and $f\left(w_{2}\right)$-testing in each case whether $C(\kappa, \lambda) / R$ is a splitting lattice, using the algorithm of Theorem 6.1. If no such $b$ as above turns up, then we are done; $w_{1}$ does not cover $w_{0}$. Otherwise we will have constructed a definite $b: C(\kappa, \lambda) \rightarrow B$ with the above properties.

In this case, $g=b f$ is bounded (Theorem 5.1), and we continue by constructing the upper and lower limits for $g, \alpha$ and $\beta$, using Lemma 6.1. Finally, after all the preliminaries we will have $w_{0}<w_{1}$ iff simultaneously $g\left(w_{0}\right)<g\left(w_{1}\right), w_{1}$. $\alpha g\left(w_{0}\right)=w_{0}$ and $w_{0}+\beta g\left(w_{1}\right)=w_{1}$. Indeed, the necessity of these conditions is quite clear $\left(g\left(w_{1} \cdot \alpha g\left(w_{0}\right)\right)=g\left(w_{0}\right)\right.$ giving $w_{0} \leq w_{1} \cdot \alpha g\left(w_{0}\right)<w_{1}$, etc. $)$. For the sufficiency, assume that $w_{0}<w \leq w_{1}, w \in|F L(\kappa)|$, and the three conditions are 
valid. Then either $g(w)=g\left(w_{0}\right)$ or $g(w)=g\left(w_{1}\right)$. If the former, then $w \leq \alpha g\left(w_{0}\right)$ and $w \leq w_{1} \cdot \alpha g\left(w_{0}\right)=w_{0}$, a contradiction. Thus $g(w)=g\left(w_{1}\right), \beta g\left(w_{1}\right) \leq w$; and we get $w=w_{1}$.

One-half of the second assertion of the next theorem was proved by B. Jónsson, using essentially the argument given below; see Remark 6.1.

Theorem 6.3. Finite sublattices of free lattices form a recursive class. This class consists of the lattices $B \in \mathbb{B}$ which satisfy Whitman's (W2).

Proof. The first statement follows from the second by Theorem 6.1. One-half of the second statement was proved in $\$ 5$. To get the other half, assume that $B$ belongs to $B$ and satisfies (W2). We must construct an embedding of $B$ into FL $(\omega)$. Denote by $\xi$ the cardinality of $B$ and set $\lambda=2^{\xi \cdot \xi}$. Choose $f: \operatorname{FL}(\xi) \rightarrow B$ and construct $f^{-}$from a table of limits.

Now construct functions $\beta_{\mu}(\mu \leq \lambda)$ employing the same iterative procedure used to generate the lower half of the standard table of limits of $B$; but beginning with $\beta_{0}=f^{-}$. Then, whenever $\mu \leq \lambda$ and $b \in|B|$, we shall have $f \beta_{\mu}(b)=b$; so $\beta_{\lambda}$ is one-to-one. We now assert that $\beta_{\lambda}$ is the desired embedding.

First, observe that $\beta_{\lambda}$ must be additive; because, by Lemma 6.1 , the $\lambda_{\text {th }}$ lower approximant in the standard table of $B$ is an additive function, and this function can be translated into $\beta_{\lambda}$ by composing with an appropriate endomorphism of $\operatorname{FL}(\xi)$. Finally, and decisively, $\beta_{0}=f^{-}$is multiplicative and Jónsson observed that, since $B$ satisfies (W2), this multiplicativity is inherited by all the functions $\beta_{\mu}$-in particular, $\beta_{\lambda}$ is multiplicative. The inductive proof of Jónsson's observation is left to the reader.

Remark 6.3. The concept of a projective object in a category is well known. A lattice $P$ is "projective" (in the category of lattices) iff every lattice homomorphism $g: A \rightarrow P$ is split by some homomorphism $f: P \mapsto A$, i.e. $g f$ is the identity function of $P$. An equivalent condition is that some homomorphism $F \rightarrow P$ splits, where $F$ is a free lattice. A. Kostinsky has supplied an entirely different proof for Theorem 6.3 which proves a more general result: A finitely generated lattice $B$ can be embedded into $\mathrm{FL}(\omega)$ iff $B$ is a bounded image of a free lattice and, in addition, satisfies (W2); and also yields: Every finitely generated sublattice of a free lattice is projective. [The history of these two results is actually rather com. plicated; without going into details, they should be attributed to Kostinsky, Jónsson and the author.]

Kostinsky's argument combines various ideas of this paper, in particular the proof of Lemma 5.2. Assume that $F$ is a finitely generated free lattice and that $g: F \rightarrow B$ is a bounded epimorphism. Let $\alpha$ and $\beta$ be the upper and lower limit functions for $g$, and let $\sigma$ be the endomorphism of $F$ which coincides with the function $\alpha g$ on the generators of $F$. Obviously, the map $b=\sigma \beta$ is a section of $g$, $g h=1_{B}$. Kostinsky shows that $b$ is a monomorphism $B \mapsto F$ iff $B$ satisfies (W2). 


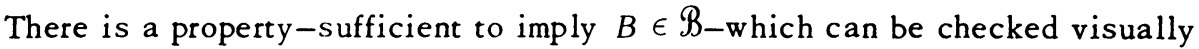
on a diagram of $B$, without recourse to pencil and paper (as opposed to the method of limit tables). To define this property, we reintroduce the sets $M(B, b)$ from the construction of a standard table, and the relation between sets of a lattice- $U \ll V-$ used to prove Lemma 6.1. Now let $M^{0}(B)=0$-the empty set-and in general, for $x \in|B|$, let

$$
x \in M^{\lambda+1}(B) \Leftrightarrow \forall U \in M(B, x)\left\{U \ll\{x\} \Rightarrow \exists V \in M(B, x)\left[U \ll V \& V \subseteq M^{\lambda}(B)\right]\right\} .
$$

A system of sets $S^{\lambda}(B)$ is defined by dual considerations. Clearly these are increasing sequences, constant after $\kappa$ terms if $|B|=\kappa$.

Theorem 6.4 (B. Jónsson). Assume that $B$ is a finite lattice and $\lambda<\omega$. If $M^{\lambda}(B)=|B|$, then the upper standard limit table for $B$ closes on or before the $(\lambda+1)$ th column. If also $S^{\lambda}(B)=|B|$ then $B \in \mathfrak{B}$.

The proof of this theorem is by induction, showing that if $\mu<\omega$, then $\alpha_{\mu}(x)=$ $a_{\mu+1}(x)$ in the standard table for $B$, whenever $x \in M^{\mu}(B)$. For the details, suppose that $\alpha_{\mu}$ and $\alpha_{\mu+1}$ coincide on $M^{\mu}$. Let $y \in M^{\mu+1}$ and let $U \in M(B, y)$, so that $w=\bigwedge \alpha_{\mu+1} U$ is a typical summand of $\alpha_{\mu+2}(y)$. If $U \ll\{y\}$, i.e. if $u_{0} \leq y$ for some $u_{0} \in U$, then $w \leq a_{\mu+1}(y)$. Otherwise, we have a set $V \in M(B, y)$ satisfying $V \subseteq M^{\mu}$ and $U \ll V$ (since $y \in M^{\mu+1}$ ). Then

$$
w \leq \wedge a_{\mu+1} V=\wedge a_{\mu} V \leq a_{\mu+1}(y)
$$

since $a_{\mu}=a_{\mu+1}$ on $V$. Summing over similar relations then gives $a_{\mu+2}(y) \leq$ $\alpha_{\mu+1}(y)$, and the rest is trivial.

There is a similar property, at least as strong, which implies that $B \in \mathcal{B}$ via the special tables. Here we put

$$
\begin{aligned}
& M_{0}^{\lambda+1}(B)=\left\{x \in|B|: \cup M_{0}(B, x) \subseteq M_{0}^{\lambda}(B)\right\}, \\
& S_{0}^{\lambda+1}(B)=\left\{x \in|B|: \cup S_{0}(B, x) \subseteq S_{0}^{\lambda}(B)\right\} ;
\end{aligned}
$$

and the condition is that, for some $\lambda, M_{0}^{\lambda}=S_{0}^{\lambda}=|B|$.

Examples of $\mathcal{B}$ lattices failing either of the se conditions have not been found. In fact the whole problem of finding a good intrinsic characterization for $\mathbb{B}$, or for the finite sublattices of a free lattice, is still open (see Problem 5 and the following).

Problem 7 (B. Jónsson). For $B \in \mathfrak{B}$, must $M^{\lambda}(B)=S^{\lambda}(B)=|B|$ for some $\lambda$ ?

Discussion. One may well ask what happens to the results of the last four sections when the role played by $\Lambda$, implicitly in that discussion, is assumed by some larger theory $\Theta$. We should then consider the splittings of an interval sublattice of $\mathfrak{T}_{l},[\Theta, \Omega]$. An attempt to extend the comprehensive theory of $\S_{3-5}$ 
to this case has led more to unsolved problems than to new results. However, the limit tables of this section have found some interesting applications in the more general situation (e.g. in Examples 7.1, 7.8 and 7.11 below).

To get much information about the splittings of proper intervals of $\mathfrak{T}_{l}$, new ideas will surely be needed. Nevertheless, given $\Theta$, one might attempt to proceed by direct analogy with what we have done, only considering theories containing $\Theta$, replacing arbitrary lattices in those considerations by lattices of $\mathcal{O} \Theta$, and using the $\Theta$-free lattices $F L(\Theta, \kappa)$ in place of absolutely free lattices. It remains true that every splitting pair of $[\Theta, \Omega]$ is of the form $\left\langle\Theta_{l}[\Theta \cup\{\epsilon\}], \Theta L\right\rangle$ where $L$ is SI and belongs to $\mathcal{\Theta}$. We might call $\epsilon, L$ a conjugate pair over $\Theta$-their characteristic property is that every $\Gamma \geq \Theta$ satisfies just one of the conditions $\epsilon \in \Gamma$, $L \in \mathcal{O} \Gamma$-and we will say that $L$ is a splitting lattice over $\Theta$. But it does not seem likely that every splitting of the interval will determine such a lattice uniquely. The concept of bounded homomorphism remains the same. With these changes, many of the results of $\$ \S 3-5$ become questionable.

For example, setting $\Theta=M$, we ask: Does every equation $\epsilon \notin \mathrm{M}$ fail in some finite modular lattice (a problem of Jónss on [6])? Is every splitting of $[M, \Omega]$ effected by a finite lattice? By a bounded epimorph of a free modular lattice? By an atomic quotient in a free modular lattice? (As in Theorem 5.4, every atomic quotient in a $\mathrm{FL}(\mathrm{M}, \kappa)$ causes a splitting.) If $g: A \rightarrow \mathrm{FL}(\mathrm{M}, \kappa)$, where $A$ is a finitely generated modular lattice, must $g$ be bounded?

Nonetheless, it is highly significant that the analogue of Lemma 5.1 is true in full generality. Repeating the proof of Theorem 5.1, part I, we therefore have the following result, where $\Theta$ is arbitrary: Assume that $g: F L(\Theta, \kappa) \rightarrow L$ is bounded, and that $L$ is finite and SI (or, more generally, that $L$ bas an atomic quotient collapsed by every proper homomorpbism of $L$ ). Then $L$ is a splitting lattice over $\Theta$, and a conjugate equation for $L$ can be obtained directly from the limits of $g$. In fact, just as before, the equation can be chosen so that it induces an atomic quotient in $\mathrm{FL}(\Theta, \kappa)$, and so that $g$ is the "largest" homomorphism of $\mathrm{FL}(\Theta, \kappa)$ separating the ends of the quotient.

Thus far, limit tables were only constructed for homomorphisms of a free lattice, but they can obviously be employed in exactly the same way to find limits and conjugate equations in this broader context. (Remark 6.2 is still relevant in this case.) Let us observe that, having constructed a special table for $f: \mathrm{FL}(\kappa) \rightarrow$ $L$, ( $L$ finite) we can use the same table for the corresponding map $g: F L(\Theta, \kappa) \rightarrow$ $L$, if $L \in \mathcal{O} \Theta$. We simply factor $f=g b$ and think of the tabular entries $w$ as representing the corresponding elements $b(w)$. The table may fail to close (over $\Lambda$ ) and yet close over $\Theta$-i.e., $g$ may be bounded when $f$ is not; in fact the greatest significance of these methods probably derives from this possibility (see, in particular, Examples 7.8 and 7.11 ). In the general case, discovering the consequences of a 
table becomes less mechanical than when $\Theta=\Lambda$-because we have no analogue of Lemma 6.1, and because we may have no effective way of testing whether two columns are equal in $F L(\Theta, \kappa)$.

7. Examples. In the last section we saw that certain questions are "decidable" in the mathematical sense of the word. In this section we find that the methods involved are sufficiently efficient to be useful in concrete applications. Most of the facts obtained and stored here are needed elsewhere in the paper.

The lattices of Diagrams $1-12$ are all subdirectly irreducible. $Q_{0}-Q_{4}$, all the $N_{\mu}$, and the lattice in Diagram 12 are splitting lattices, whereas no homomorphism from a free lattice onto $M_{3}, P_{0}$ or $P_{1}$ is either upper or lower bounded (proof of Corollary 5.3). $Q^{*}$ is not a splitting lattice but it is a lower bounded epimorph of $\mathrm{FL}(3)$. These remarks are justified below. One concludes from Theorem 6.3 , by inspection of the diagrams, that $N_{\mu}$ and $Q_{0}-Q_{4}$ have isomorphic copies in FL $(\omega)$ and the other lattices do not.

Each table accompanying an example below is a special limit table, constructed according to the specifications of $\$ 6$, for a homomorphism of a free lattice which replaces the free generators, denoted by boldface letters in the table, by the generators of the given lattice, designated by corresponding light face letters in its diagram. Tables not given can be easily supplied by the reader. It is not necessary to enter rows for the 0 and 1 of the lattice unless the limit words of these elements are required, because they do not enter into the construction of the other rows (review the definition). Rather than make two successive identical entries in a row we always mark the second with "*".

Example 7.1 (Diagram 1 and Table 1). The table for $M_{3}$ of course cannot close. The first two equations in a discriminating system (Remark 6.2) for $M_{3}$ are the distributive law and the equation

$$
x \cdot[y \cdot(x+z)+z \cdot(x+y)] \leq y+(x+y z) \cdot(z+x y),
$$

both obtained from the lower $a$ and upper $b$ rows. But when we relativize the table to modular theory, $M$ (as in the Discussion $\$ 6, \mathbb{1} 5$ ), it closes with the second column, proving the well-known fact that $M_{3}$ and the distributive law are conjugate over M.

Example 7.2. Limit tables for $N_{5}, P_{0}$ and $P_{1}$ are left to the reader. The infinite sets of discriminating equations that will be obtained for $P_{0}$ and $P_{1}$ have an interesting pattern. The table for $N_{5}$ closes in two columns and yields the modular law.

Example 7.3 (Diagram 5 and Table 2). The limit table for $Q_{0}$ closes in two columns, and the conjugate equation, computed from the limits of $b$ and $(a+b)$. $(b+c)$ displayed in column two, is

$\zeta_{0} \cdot[x+y \cdot(z+x y)] \cdot(z+x y) \leq y+[x+z \cdot(x+y)] \cdot(y+z)$. 
Example 7.4 (Diagram 6). The limit table for $Q_{1}$ closes in three columns and the conjugate equation simplifies to

$\zeta_{1} \cdot x \cdot\{x y+z \cdot(w+x y z)\} \leq x y+(z+w) \cdot[x+w \cdot(x+z)]$.

Example 7.5 (Diagram 7). The conjugate equation of $Q_{2}$ is

$\zeta_{2} \cdot(x+y) \cdot(x+z)=x+(x+y) \cdot(x+z) \cdot(y+z)$.

Example 7.6 (Diagram 8). The conjugate equation of $Q_{3}$ is

$\zeta_{3} \cdot(x+y z) \cdot(z+x y)=z \cdot(x+y z)+x \cdot(z+x y)$.

Example 7.7 (Diagram 9). A limit table for $Q_{4}$ closes in three columns giving a conjugate equation we have been unable to simplify:

$\zeta_{4} \cdot y \cdot\{z+y \cdot(x+y z)\} \leq x+(x+y) \cdot[z+x \cdot(y+z)]$.

Example 7.8 (Diagram 10 and Table 3). By Corollary 5.3, the upper limit table for $Q^{*}$ cannot close and this is borne out by Table 3 . However, since $Q_{4} \notin \mathbf{H S}\left(Q^{*}\right)$, by Jónsson's lemma $Q_{4} \notin \mathcal{O} Q^{*}$; and hence $Q^{*}$ satisfies $\zeta_{4}$, the conjugate of $Q_{4}$ (Example 7.7). If we put $\Theta=\Theta_{l}\left[\zeta_{4}\right]$, then it is easy to see that the second and third columns of Table 3 become identical in $\operatorname{FL}(\Theta, 3)$.

In fact, replacing $y$ by $(x+y) \cdot(y+z)$ in $\zeta_{4}$ gives that

$$
(x+y) \cdot\{z+(y+z) \cdot[x+z \cdot(x+y)]\} \leq_{\theta} x+(x+y) \cdot[z+x \cdot(y+z)],
$$

which certainly implies that $\alpha_{2}(a)=\alpha_{1}(a)$ and $\alpha_{2}(c)=\alpha_{1}(c)$ in $\operatorname{FL}(\Theta, 3)$.

Therefore (Discussion $\$ 6) Q^{*}$ is a splitting lattice over $\Theta$. The conjugate of $Q^{*}$ over $\Theta$, obtained from the entries $\beta_{1}(a+b)$ and $\alpha_{1}(a)$ in Table 3 , is equivalent to the equation

$\zeta_{5} \cdot y \cdot(x+z) \leq x+(x+y) \cdot[z+x \cdot(y+z)]$.

To summarize: If $\Theta \in \mathbf{T}_{l}$, then $\zeta_{4} \in \Theta$ iff $\Theta \not 囚 Q_{4}$; and if $\zeta_{4} \in \Theta$, then $\zeta_{5} \in \Theta$ iff $\Theta \nless \Theta Q^{*}$.

Example 7.9 (Diagram 11). A limit table for $N_{6}$ closes in three columns giving a conjugate equation which simplifies to

$\zeta_{6} \cdot y \cdot[x \cdot(w+x z)+z \cdot(w+x z)] \leq x+[x+y+w \cdot(x+z)] \cdot[z+w \cdot(x+z)]$. By Example 5.2 (or Theorem 6.4), all the $N_{\mu}$ are splitting lattices. The equations conjugate to these lattices quickly become rather complex.

Example 7.10 (Diagram 12). Since $Q$ fails Whitman's (W2) (witness $a \cdot(b+c)$ $\leq a b+c)$ it cannot be embedded in a free lattice. Nevertheless it is a splitting lattice and a limit table gives

$$
\zeta_{7} \cdot(x+y z) \cdot(y+x z) \cdot(z+x y) \leq x \cdot(y+z)+y \cdot(x+z)+z \cdot(x+y)
$$

as its conjugate equation. Incidentally, $Q$ was constructed from the free distributive lattice, $\operatorname{FL}(\Delta, 3)$, by adding the two elements represented by the terms of $\zeta_{7}$.

Example 7.11. In [7], Jónsson considered a certain eight element simple modular lattice $M_{3,3}$, and he showed, in effect, that if $\mathrm{P}$ is the theory of any infinite lattice having no four element chain, then $\left\langle P, \Theta M_{3,3}\right\rangle$ is a splitting pair for [M, $\Omega]$ (cf. the Discussion \$6). 
Now it turns out that $M_{3,3}$ is a bounded image of $F L(M, 4)$, and a limit table for this homomorphism yields an equation equivalent over $M$ to the one given by Jónsson (as a base, together with the modular law, for P).

8. Covering in $\mathfrak{T}_{l}$. In $[6$, Corollaries $4.4,4.7]$ Jónsson obtained the intriguing result that every theory $\Theta>\Lambda$ covers another the ory $H>\Lambda$-in symbols: $H<\Theta$ in $\mathfrak{T}_{l}$. When we attempt to go beyond this result to gain a better understanding of the relation of covering in $\mathfrak{F}_{l}$, a multitude of problems arise to challenge our ingenuity. In this section we will state explicitly a number of these which seem to us most interesting. Contact will be made with the theory of splittings.

We begin the discussion by observing the negative result that not every $\Theta<\Omega$ can be covered by another theory; $\Theta=\Lambda$ gives an example [6]. In fact, if $\Theta$ is a meet-irreducible theory then it will be covered (and then uniquely) iff it is strictly meet-irreducible. (Compare Theorem 4.3.) Next, note that a finitely based theory $\Theta$ covers only one theory just in case it is join-irreducible; in this case the covered theory is $\Theta . \Theta$ where $L$ is the conjugate lattice of $\Theta$. (Compare Theorem 4.2 and Corollary 5.8.)

In order to extend this result, we now introduce a convenient notation: $C_{V} \Theta$ is the set of all theories $H \in T_{l}$ such that $H<\Theta$. Recall the definition of $\nabla_{l} \Theta$ in $\S 1$.

Theorem 8.1. Assume that $\Theta$ bas a finite base and that $\Lambda<\Theta$. Then

$$
\nabla_{l} \Theta=\left\{\kappa: 1 \leq \kappa<\omega \text { and } \kappa \leq{ }^{\prime}\left(C_{V} \Theta\right)\right\} \text {. }
$$

Proof. First, assume that $\kappa \in \nabla_{l} \Theta$ and let $\left\{\epsilon_{0}, \ldots, \epsilon_{\kappa-1}\right\}$ be some $\kappa$-element independent base of $\Theta$. For each $\nu<\kappa$ choose a maximal theory $\Theta_{\nu} \leq \Theta$ from among all the subtheories of $\Theta$ which contain all equations $\epsilon_{\mu}, \mu \neq \nu$, but not $\epsilon_{\nu}$. Then of course we have $\Theta_{\nu}<\Theta$ whenever $\nu<\kappa$, and $\Theta_{\nu}+\Theta_{\nu^{\prime}}=\Theta$ whenever $\nu \neq \nu^{\prime}$. Hence $\kappa \leq \notin\left(C_{V} \Theta\right)$.

Next, assume that $1<\kappa<\omega$ and $\kappa \leq \#\left(C_{V} \Theta\right)$. (We already know that $1 \epsilon$ $\nabla_{l} \Theta$ by Lemma 1.2(ii).) Choose any $\kappa$ theories covered by $\Theta$, say $\Theta_{0}, \ldots, \Theta_{\kappa-1}$. For $\nu<\kappa$, set

$$
\Gamma_{\nu}=\bigwedge\left\{\Theta_{\mu}: \mu \neq \nu\right\}
$$

Then, since $\mathscr{T}_{l}$ is distributive, we have

$$
\Theta=\bigwedge\left\{\Theta_{\mu}+\Theta_{\nu}: \mu \neq \nu\right\}=\bigvee\left\{\Gamma_{\nu}: \nu<\kappa\right\}
$$

Now, because $\Theta$ is finitely based, the above relation yields the existence of a system of compact (finitely based) theories $\mathrm{K}_{\nu} \leq \Gamma_{\nu}$ such that $\Theta=\mathrm{V}\left\{\mathrm{K}_{\nu}\right\}$. By Lemma 1.2(ii), this leads to a system of equations $\epsilon_{\nu} \in \Gamma_{\nu}$ such that $\Theta=$ $\Theta_{l}\left[\epsilon_{0}, \cdots, \epsilon_{\kappa-1}\right]$. Finally, since 


$$
\left\{\epsilon_{\mu}: \mu \neq \nu\right\} \subseteq \bigvee\left\{\Gamma_{\mu}: \mu \neq \nu\right\} \leq \Theta_{\nu}
$$

whenever $\nu<\kappa,\left\{\epsilon_{\nu}\right\}$ is an independent base of $\Theta$; and we have $\kappa \in \nabla_{l} \Theta$. (Here we have given the proof of Lemma 1.3.) The theorem is proved.

If now $\Theta$ is an arbitrary theory having an independent base of $\kappa$ equations, where $\kappa \leq \omega$, then we can still assert the existence of at least $\kappa$ theories covered by $\Theta$, and the proof is just the same as above. But, besides these facts, the author has been unable to establish essentially any other relationships between $\nabla_{l}$ and $C_{V}$. Even worse, we do not know what values these functions can assume.

Many of the problems that come to mind in this connection are included in the following scheme. First we list several conditions on the theory $\Theta$ :

(1) $\Theta=\Theta L$ for some finite lattice $L$.

(2) $\Theta=(L$ for some splitting lattice $L$, i.e. $\Theta$ is strictly meet-prime.

(3) ${ }_{\kappa}(1 \leq \kappa<\omega) \nabla_{l} \Theta=[1, \kappa]$.

(4) $\nabla_{l} \Theta=[1, \omega)$.

(5) $\nabla_{l} \Theta=0$.

(6) $\nabla, \Theta=\{\omega\}$

By Lemma 1.2, every lattice theory other than $\Lambda$ satisfies just one of the conditions (3) through (6).

Now we ask: For each of the above conditions, how does it restrict the number and the nature of the theories covered by $\Theta$ ? Let me give two of these problems a more definite formulation.

Problem $2^{\circ}$. If $\mathrm{H}<\boldsymbol{\Theta}, L$ finite, does $\mathbf{H}=\boldsymbol{\Theta} L^{\prime}$ for some finite $L^{\prime}$ ?

(We can, analogously, ask whether compactness is a hereditary property of theories under the covering relation.)

Problem 8. If $\Theta=\Theta L$, $L$ finite, must we have $\sharp\left(C_{V} \Theta\right)<\boldsymbol{\kappa}_{0}$ ? or $\leq \boldsymbol{\kappa}_{0}$ ?

(Again we can ask the same question under the weaker assumption that $\Theta$ is compact, or under the stronger assumption of (2).)

A negative solution of either of these problems would be of great interest. A positive solution of both would add some impetus to the attempt to actually describe $C_{V} L$ when $L$ is finite.

This latter task has been accomplished for $M_{3}$ ([4] and [7]), while the author's attempt to do it for $N_{5}$ led to the present paper. It is very much an open question whether a sufficient knowledge of the splittings of $\mathfrak{T}_{l}$ and its intervals would be adequate to handle all specific problems of this sort. But, to demonstrate this possibility, we will now derive the known facts about $C_{V} \boldsymbol{\Theta M}_{3}$ and $C_{V} \Theta N_{5}$ from the existence of certain splittings. The (incomplete) results for $N_{5}$ were obtained by the author and, in part, by Stephen Comer at Vanderbilt, and by Mr. Lee, Bruns' student at McMaster.

From [7], the members of $C_{V} \Theta M_{3}$ are the theories of three finite lattices: $M_{3} \times N_{5}$, and those which he called $M_{4}$ and $M_{3,3}$. Now $\left\langle\mathbf{M}, N_{5}\right\rangle$ splits $\mathfrak{T}_{2}$; 
$\left\langle\mathrm{P}, \Theta M_{3,3}\right\rangle$ splits $[\mathrm{M}, \Omega]$ (Example 7.11); and the interval $[\mathrm{P}, \Omega]$ is a chain, ordered like $1+\omega^{*}$, two consecutive terms of which are $\Theta M_{3}$ and $\Theta M_{4}$-whence $\left\langle\Theta_{3}, \Theta_{4}\right\rangle$ splits $[P, \Omega]$. Using these facts, we can argue as follows: Suppose that $\mathrm{H} \prec \Theta M_{3}$. Then if $\mathrm{H} \leq \Theta N_{5}$, we must have $\mathrm{H}=\Theta M_{3} \cdot \Theta N_{5}\left(=\Theta M_{3} \times N_{5}\right)$; otherwise $\mathrm{M} \leq \mathrm{H}$. Then if $\mathrm{H} \leq \mathrm{OM}_{3,3}$, we get $\mathrm{H}=\boldsymbol{\Theta} M_{3,3}$; otherwise $\mathrm{P} \leq \mathrm{H}$. In this last case, either $\mathrm{H} \leq \boldsymbol{\Theta} M_{4}$ or $\Theta M_{3} \leq \mathrm{H}$; and we can only have $\mathrm{H}=\boldsymbol{\Theta}_{4}$. From this argument, the only theories possibly covered by $\Theta M_{3}$ are the three listed, and of course it was easy to see that $\Theta M_{3}$ covers each of the three theories.

Turning now to $N_{5}$, we clearly have $M_{3} \cdot N_{5}<N_{5}$ (because $\mathfrak{T}_{l}$ is distributive and $\left.\Theta M_{3}<\Delta\right)$. And trivial algebraic considerations deriving from Jónsson's lemma show that, for each lattice $L$ among $P_{0}, P_{1}, Q_{0}-Q_{4}, Q^{*}, N_{6}$ and their duals (diagrams of this paper), we have $L<N_{5}$. In fact, each of these SI lattices embeds $N_{5}$ as proper sublattice, and one simply verifies that every smaller SI lattice in HS $L$ belongs to HS $N_{5}$. Altogether, then, we have found sixteen distinct theories covered by $\Theta N_{5}$.

Problem 9. Does $C_{V} \Theta N_{5}$ include any theories besides the sixteen catalogued above?

Having found these sixteen theories one can draw considerable aid, for the further resolution of this problem, from the facts developed earlier in this paper. The relevant splittings were calculated in $\$ 7$.

Indeed, assume that we have a theory $\mathrm{H}<\boldsymbol{\Theta}_{5}$, which contains, for each one of the lattices $M_{3}, P_{0}, P_{1}, Q_{0}-Q_{4}, Q^{*}, N_{6}$ and the ir duals, an equation which fails in this lattice. By Examples 7.3-7.9, $\mathrm{H}$ then contains each one of the irreducible equations $\zeta_{0}-\zeta_{4}$ and their duals, the (reducible) equation $\zeta_{5}$ and its dual; and also equation $\zeta_{6}$.

If we let $\mathrm{Z}_{0}$ denote the theory based on all these equations, then $\mathrm{Z}_{0} \leq \mathrm{H}$. Moreover, for each one of the lattices $B=M_{3}, P_{0}, P_{0}^{d}$ or $P_{1}, \mathrm{H}$ contains one equation from any discriminating system of equations computed from a limit table for $B$. (We are sadly unable to decide whether any of these tables close over $\mathrm{Z}_{0}$; cf. the Discussion $\$ 6$. ) In this manner, we find that Problem 9 reduces to the question whether, with every choice of equations $\epsilon_{0}, \cdots, \epsilon_{3}$ from these discriminating systems, the two equations $\eta_{1}$ and $\eta_{2}\left(\S_{2}\right)$ can be derived from $\epsilon_{0}, \ldots, \epsilon_{3}$ combined with $\mathrm{Z}_{0}$.

Let us return briefly to the question of the existence of theories satisfying conditions (3)-(6) above. In [9] it was shown that condition (6) is nonvacuous. We have no examples of lattice theories satisfying (4), (5), or (3) $)_{K}$ for any $\kappa>12$. However, by Lemma 1.2, Theorem 8.1 and the above, $\Theta N_{5}$ satisfies either (4) or $(3)_{\kappa}$ for some $\kappa \geq 16$. For the the ory $\mathrm{Z}_{0}$ defined above, $\nabla_{l} \mathrm{Z}_{0}=[1,12]$. And one obtains from $\mathrm{Z}_{0}$ theories satisfying (3) through (3) $)_{11}$ by subtracting the equations $\zeta$ from its generating set, one at a time, beginning with $\zeta_{5}$ and then $\zeta_{5}^{d}$. For 


\begin{tabular}{l|c|c|c|l} 
& $a_{0}$ & $a_{1}$ & $a_{2}$ & $\ldots$ \\
$M_{3}$ & $\beta_{0}$ & $\beta_{1}$ & $\beta_{2}$ & \\
\hline$a$ & $\mathbf{a}$ & $\mathbf{a}+\mathbf{b c}$ & $\mathbf{a}+(\mathbf{b}+\mathbf{a c}) \cdot(\mathbf{c}+\mathbf{a b})$ & \\
& $\mathbf{a}$ & $\mathbf{a} \cdot(\mathbf{b}+\mathbf{c})$ & $\mathbf{a} \cdot[\mathbf{b} \cdot(\mathbf{a}+\mathbf{c})+\mathbf{c} \cdot(\mathbf{a}+\mathbf{b})]$ & $\cdots$ \\
\hline$b$ & $\mathbf{b}$ & $\mathbf{b}+\mathbf{a c}$ & $\mathbf{b}+(\mathbf{a}+\mathbf{b c}) \cdot(\mathbf{c}+\mathbf{a b})$ & $\ldots$ \\
& $\mathbf{b}$ & $\mathbf{b} \cdot(\mathbf{a}+\mathbf{c})$ & $\mathbf{b} \cdot[\mathbf{a} \cdot(\mathbf{b}+\mathbf{c})+\mathbf{c} \cdot(\mathbf{a}+\mathbf{b})]$ & \\
\hline & $\mathbf{c}$ & $\mathbf{c}+\mathbf{a b}$ & $\mathbf{c}+(\mathbf{a}+\mathbf{b c}) \cdot(\mathbf{b}+\mathbf{a c})$ & $\ldots$ \\
& $\mathbf{c}$ & $\mathbf{c} \cdot(\mathbf{a}+\mathbf{b})$ & $\mathbf{c} \cdot[\mathbf{a} \cdot(\mathbf{b}+\mathbf{c})+\mathbf{b} \cdot(\mathbf{a}+\mathbf{c})]$ & \\
\hline
\end{tabular}

Table 1

\begin{tabular}{|c|c|c|c|}
\hline$a$ & $\begin{array}{l}a+c \cdot(a+b) \\
a\end{array}$ & $\begin{array}{l}* \\
*\end{array}$ & * \\
\hline$b$ & $\begin{array}{l}\mathbf{b}+\mathbf{a} \cdot(\mathbf{b}+\mathbf{c})+\mathbf{c} \cdot(\mathbf{a}+\mathbf{b}) \\
\mathbf{b} \cdot(\mathbf{c}+\mathbf{a b})\end{array}$ & $\begin{array}{c}\mathbf{b}+[\mathbf{a}+\mathbf{c} \cdot(\mathbf{a}+\mathbf{b})] \cdot(\mathbf{b}+\mathbf{c}) \\
*\end{array}$ & * \\
\hline$c$ & c & * & $*$ \\
\hline$a+b$ & $\begin{array}{l}a+b \\
(a+b) \cdot(a+c)\end{array}$ & $\begin{array}{c}* \\
\mathbf{a}+\mathbf{b} \cdot(\mathbf{c}+\mathbf{a b})\end{array}$ & $*$ \\
\hline$b+c$ & $\begin{array}{l}\mathbf{b}+\mathbf{c} \\
\mathbf{c}+\mathbf{a b}\end{array}$ & $\begin{array}{l}* \\
*\end{array}$ & $*$ \\
\hline$(a+b) \cdot(b+c)$ & $\begin{array}{l}(a+b) \cdot(b+c) \\
(a+b) \cdot(c+a b)\end{array}$ & $\begin{array}{c}* \\
{[a+b \cdot(c+a b)] \cdot(c+a b)}\end{array}$ & $*$ \\
\hline$a b$ & $\begin{array}{l}a \cdot(b+c)+c \cdot(a+b) \\
a b\end{array}$ & $\begin{array}{c}{[a+c \cdot(a+b] \cdot(b+c)} \\
*\end{array}$ & $*$ \\
\hline
\end{tabular}

Table 2 
$1 \leq \kappa \leq 10$, this follows from the fact that the theory in question has an independent base of $\kappa$ irreducible equations. (It is not true, conversely, that if $\nabla_{l} \Theta=$ $[1, \kappa]$ then $\Theta$ has a base of irreducible equations; see Corollary 5.6.) The following problem, deferred from $\$ 4$ and $\$ 5$, is clearly relevant here.

Problem 10. Describe the ordered structure of join-irreducible theories. Or at least determine the possible cardinalities of independent sets of irreducible equations.

We conclude with a question whose positive solution is not expected, even though it would make some of the problems of this section much easier; it concerns a possible extension of Jónsson's result with which we opened the section. The answer is obviously yes if $\Theta$ is a compact theory.

Problem 11. Given $\Gamma<\Theta$, must there exist a theory $H$ such that $\Gamma \leq H<\Theta$ ?

If the answer to this problem were positive, we could prove, for example, that ${ }^{F}\left(C_{V} \Theta\right) \leq \kappa_{0} \Rightarrow \nabla_{l} \Theta \neq 0$.

\begin{tabular}{|c|c|c|c|c|}
\hline$Q^{*}$ & $\begin{array}{l}a_{0} \\
\beta_{0}\end{array}$ & $\begin{array}{l}\alpha_{1} \\
\beta_{1}\end{array}$ & $\begin{array}{l}\alpha_{2} \\
\beta_{2}\end{array}$ & \\
\hline$a$ & $\begin{array}{c}\mathbf{a}+\mathbf{c} \cdot(\mathbf{a}+\mathbf{b}) \\
\mathbf{a}\end{array}$ & $\begin{array}{c}a+\{c+a \cdot(b+c)\} \cdot(a+b) \\
*\end{array}$ & $\begin{array}{c}\mathbf{a}+\alpha_{1}(c) \cdot(\mathbf{a}+\mathbf{b}) \\
*\end{array}$ & $m$ \\
\hline$b$ & $\begin{array}{c}(a+b) \cdot(b+c) \\
b \cdot(a+c)\end{array}$ & * & * & \\
\hline$c$ & $\begin{array}{c}\mathbf{c}+\mathbf{a} \cdot(\mathbf{b}+\mathbf{c}) \\
\mathbf{c}\end{array}$ & $\begin{array}{c}c+\{a+c \cdot(a+b)\} \cdot(b+c) \\
*\end{array}$ & $\begin{array}{c}\mathbf{c}+a_{1}(a) \cdot(\mathbf{b}+\mathbf{c}) \\
*\end{array}$ & $m$ \\
\hline$a+b$ & $\begin{array}{c}a+b \\
(a+b) \cdot(a+c)\end{array}$ & ${ }^{*} \mathbf{a}+\mathbf{b} \cdot(\mathbf{a}+\mathbf{c})$ & * & \\
\hline$b+c$ & $\begin{array}{c}b+c \\
(b+c) \cdot(a+c)\end{array}$ & $\begin{array}{c}* \\
\mathbf{c}+\mathbf{b} \cdot(\mathbf{a}+\mathbf{c})\end{array}$ & * & \\
\hline
\end{tabular}

Table 3 

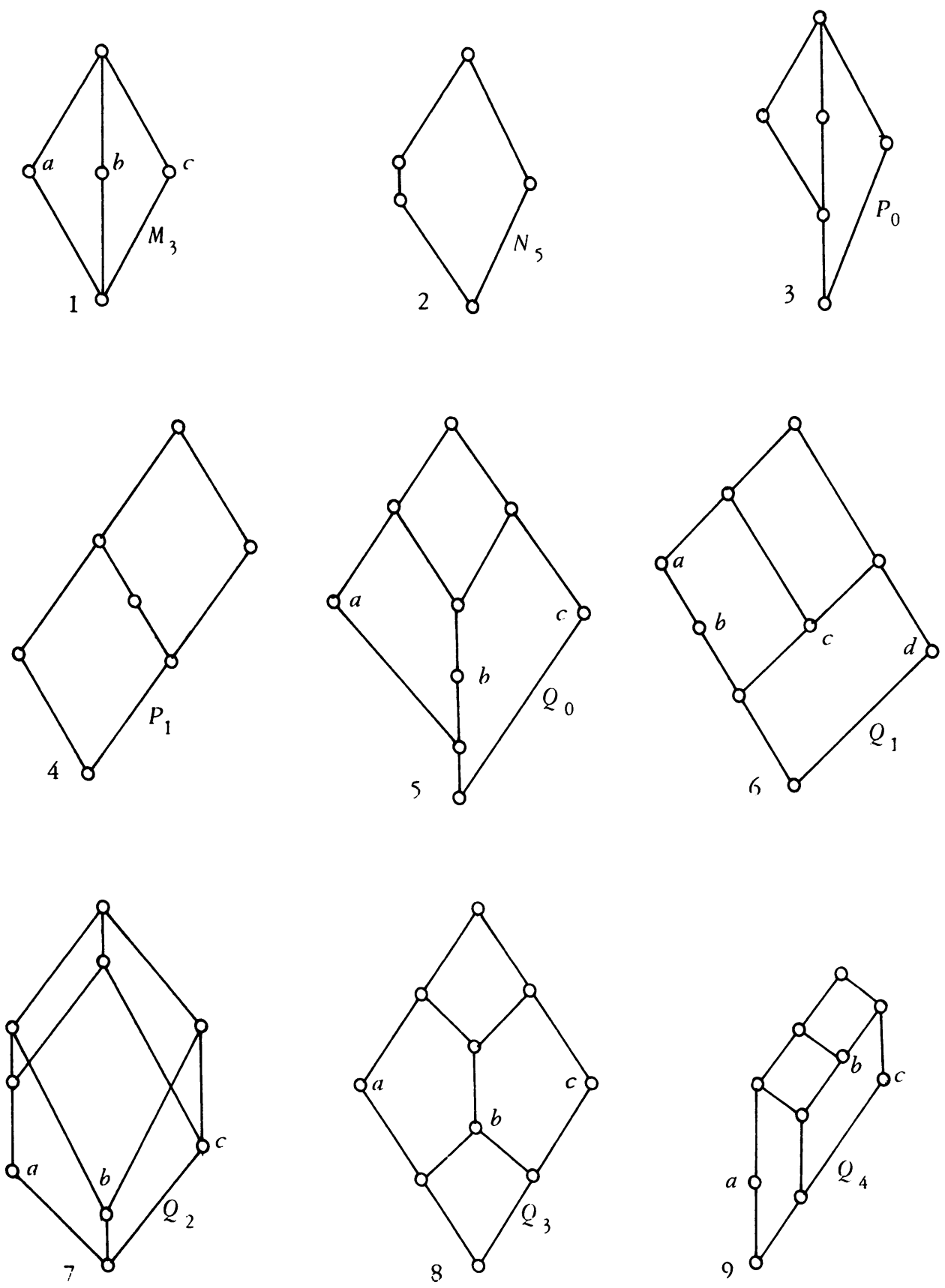

Diagrams 


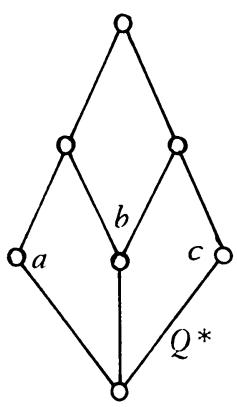

10
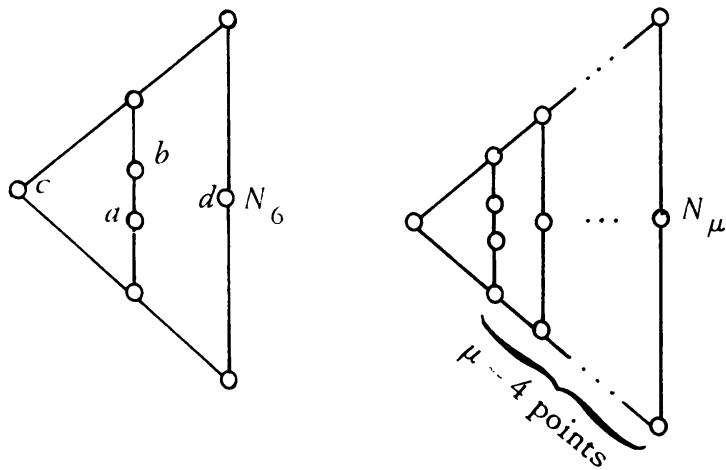

11

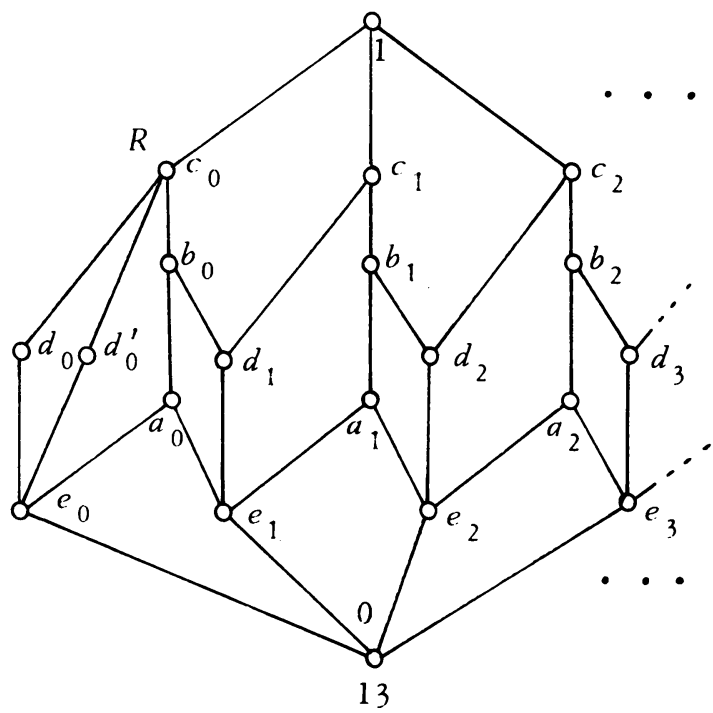

\section{Diagrams}

\section{BIBLIOGRAPHY}

1. K. A. Baker, Equational classes of modular lattices, Pacific J. Math. 28 (1969), 9-15. MR 39 \#5435.

2. G. Birkhoff, Lattice theory, 3rd ed., Amer. Math. Soc. Colloq. Publ., vol. 25, Amer. Math. Soc., Providence, R. I., 1967. MR 37 \#2638.

3. S. D. Comer and D. X. Hong, Some remarks concerning the varieties generated by the diamond and the pentagon, Trans. Amer. Math. Soc. 174 (1972), 45-54.

4. G. Grätzer, Equational classes of lattices, Duke Math. J. 33 (1966), 613-622. MR 33 \#7278.

5. - Universal algebra, Van Nostrand, Princeton, N. J., 1968. MR 40 \#1320. 
6. B. Jónsson, Algebras whose congruence lattices are distributive, Math. Scand. 21 (1967), 110-121. MR 38 \#5689.

40 \#66.

7. - Equational classes of lattices, Math. Scand. 22 (1968), 187-196. MR

8. B. Jónsson and J. E. Kiefer, Finite sublattices of a free lattice, Canad. J. Math. 14 (1962), 487-497. MR 25 \#1117.

MR 43 \#118.

9. R. McKenzie, Equational bases for lattice theories, Math. Scand. 27 (1970), 24-38.

10. M. P. Schützenberger, Sur certaines axiomes de la théorie des structures, C. R. Acad. Sci. Paris 221 (1945), 218-220. MR 7, 235.

11. A. Tarski, Equational logic and equational theories of algebras, Contributions to Math. Logic (Colloq., Hannover, 1966), North-Holland, Amsterdam, 1968, pp. 275-288.

MR $38 \# 5692$.

12. P. M. Whitman, Free lattices. I, Ann. of Math. (2) 42 (1941), 325-330. MR 2, 244.

13. - Free lattices. II, Ann. of Math. (2) 43 (1942), 104-115. MR 3, 261.

14. - Splittings of a lattice, Amer. J. Math. 65 (1943), 179-196. MR 4, 129.

15. - Status of word problems for lattices, Proc. Sympos. Pure Math., vol. 2, Amer. Math. Soc., Providence, R. I., 1961, pp. 17-21. MR 23 \#A1560.

16. R. Wille, Primitive Länge und primitive Weite bei modularen Verbänden, Math. Z. 108 (1969), 129-136. MR 39 \#2672.

DEPARTMENT OF MATHEMATICS, UNIVERSITY OF CALIFORNIA, BERKELEY, CALIFOR-

NIA 94720 